ARTICLE

Received 22 Feb 2016 | Accepted 17 Jun 2016 | Published 25 Jul 2016

DOI: $10.1038 /$ ncomms12282

OPEN

\title{
Plexins function in epithelial repair in both Drosophila and zebrafish
}

Sa Kan Yoo ${ }^{1,2,3}$, Heath G. Pascoe ${ }^{4, \star}$, Telmo Pereira ${ }^{5,6, \star}$, Shu Kondo ${ }^{7}$, Antonio Jacinto ${ }^{6}$, Xuewu Zhang ${ }^{4}$ \& Iswar K. Hariharan ${ }^{1}$

In most multicellular organisms, homeostasis is contingent upon maintaining epithelial integrity. When unanticipated insults breach epithelial barriers, dormant programmes of tissue repair are immediately activated. However, many of the mechanisms that repair damaged epithelia remain poorly characterized. Here we describe a role for Plexin A (PlexA), a protein with particularly well-characterized roles in axonal pathfinding, in the healing of damaged epithelia in Drosophila. Semaphorins, which are PlexA ligands, also regulate tissue repair. We show that Drosophila PlexA has GAP activity for the Rap1 GTPase, which is known to regulate the stability of adherens junctions. Our observations suggest that the inhibition of Rap1 activity by PlexA in damaged Drosophila epithelia allows epithelial remodelling, thus facilitating wound repair. We also demonstrate a role for Plexin A1, a zebrafish orthologue of Drosophila PlexA, in epithelial repair in zebrafish tail fins. Thus, plexins function in epithelial wound healing in diverse taxa.

\footnotetext{
${ }^{1}$ Department of Molecular and Cell Biology, University of California, Berkeley, California 94720, USA. ${ }^{2}$ The Miller Institute, University of California, Berkeley, California 94720, USA. ${ }^{3}$ Physiological Genetics Laboratory, RIKEN, 2-2-3 Minatojima-minamimachi, Chuo-ku, Kobe 650-0047, Japan. ${ }^{4}$ Department of Pharmacology, University of Texas Southwestern Medical Center, Dallas, Texas 75390, USA. ${ }^{5}$ Instituto de Medicina Molecular, Faculdade de Medicina da Universidade de Lisboa, 1649-028 Lisboa, Portugal. ${ }^{6}$ CEDOC, Chronic Diseases Research Centre, NOVA Medical School, Universidade NOVA de Lisboa, 130, 1169-056 Lisboa, Portugal. ${ }^{7}$ Genetic Strains Research Center, National Institute of Genetics, 1111 Yata, Mishima, Shizuoka 411-8540, Japan. * These authors contributed equally to this work. Correspondence and requests for materials should be addressed to S.K.Y. (email: sakan.yoo@riken.jp) or to I.K.H. (email: ikh@berkeley.edu).
} 
T he initial responses to epithelial wounds have an important role in determining whether tissue damage eventually results in regeneration or scar formation ${ }^{1-3}$. Since epithelial tissues are found in most metazoans, some of the mechanisms involved in repairing epithelial tissues and in healing wounds are likely to be evolutionarily ancient and can potentially be identified using genetic screens conducted in invertebrate model organisms ${ }^{4-7}$. We first screened for regulators of wound repair in Drosophila imaginal discs and then tested whether the orthologous genes in zebrafish have a similar function. Using this approach, we have identified a key role for plexins during tissue repair in both organisms.

\section{Results}

An RNAi screen identifies a role for PlexA in wing disc repair. The adult wing of Drosophila develops from a larval epithelial structure, the wing imaginal disc that can be visualized in situ by the nubbin $>$ GFP (green fluorescent protein) reporter and bisected by applying pressure on the larval cuticle with a tungsten needle while leaving the cuticle itself intact (Fig. 1a and Supplementary Movie 1). After wounding, the two portions of the wing disc were observed to move independently but remained in proximity to each other likely because they were attached to neighbouring structures. To examine early changes following wounding, we used a GCaMP3 probe to visualize changes in intracellular calcium ${ }^{8}$. In the vicinity of the wound, we observed a rapid increase immediately after wounding, which persisted for 5-10 min (Fig. $1 \mathrm{~b}$ and Supplementary Movie 2), which is similar to the wound-induced calcium flashes observed in the Caenorhabditis. elegans epidermis ${ }^{9}$, the zebrafish tail $\mathrm{fin}^{2}$ and the Drosophila embryonic and pupal epithelia ${ }^{10,11}$. Fusion of the two disc fragments had occurred within $6 \mathrm{~h}$ of wounding. At this time, there was an accumulation of F-actin at the site of fusion (Fig. 1c) and increased activity of the Jun N-terminal kinase (JNK) as assessed by a transcriptional reporter of AP-1 activity ${ }^{12}$
(Fig. 1d). Calcium signalling, accumulation of F-actin and the increased activity of JNK are likely to be evolutionarily ancient responses to wounding since they are found in diverse organisms. Thus, screens for regulators of wound healing in imaginal discs are likely to identify mechanisms that are conserved among diverse taxa.

Approximately 95\% of flies survive when one of their wing discs has been bisected (Fig. 1e,f). In these survivors, rejoining of the two fragments is highly efficient and $\sim 95 \%$ of the discs develop into adult wings that either look completely normal or have minor notching of the wing margin. In the remaining $5 \%$ of the adult survivors, there are a variety of defects, most notably blisters, which are often seen when the two surfaces of the adult wing fail to adhere properly.

To test whether a gene has a role in wound healing, we manipulated its activity in the wing pouch and fragmented one of the two wing discs; the intact contralateral disc served as a control (Fig. 1g). A dominant-negative form of the JNK (JNK ${ }^{\mathrm{DN}}$ ) elicited an increase in the frequency of wing morphology defects on the wounded side ( $43 \%$ versus $5 \%$ in controls; Fig. 1 h) consistent with previous observations in other systems that indicated a role for JNK in wound healing ${ }^{4,5,13-15}$. We then screened 1,193 RNA interference (RNAi) lines targeting proteins that are predicted to be either on the cell surface or secreted (CSS) ${ }^{16}$ because these CSS molecules are likely to play important roles in cell-cell communication and tissue remodelling during wound repair. We wounded at least 25 larvae per stock, thus screening $\sim 30,000$ flies (Fig. 1h). Of these, 34 lines (2.8\%) were classified as 'strong hits' since they elicited wing abnormalities only on the wounded side. Plexin A (PlexA) was the top hit, with $\sim 80 \%$ of wounded animals exhibiting wing abnormalities with only minor effects in the absence of wounding, a result confirmed using three additional RNAi lines (Fig. 1i,j and Supplementary Fig. 1a,b). In addition, to inactivate PlexA function in the wing disc in a way that does not depend on RNAi, we expressed the Cas9 nuclease and a guide RNA (gRNA) that targeted PlexA in cells of the wing a

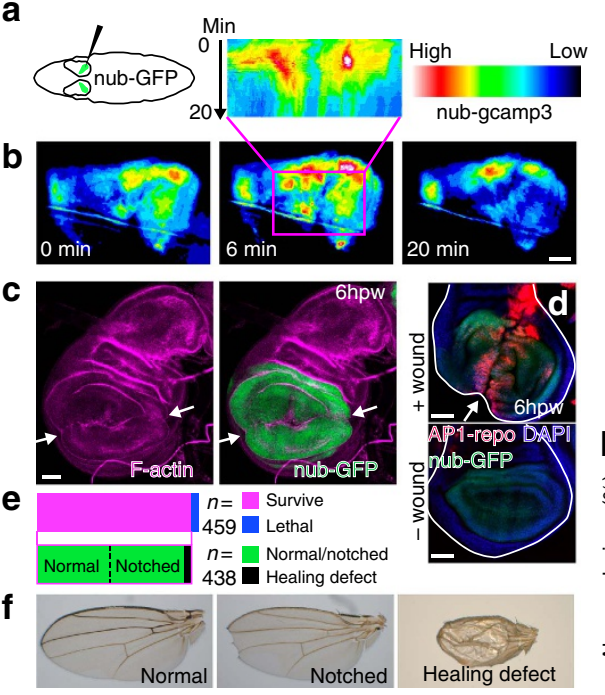

g UAS-dcr2;

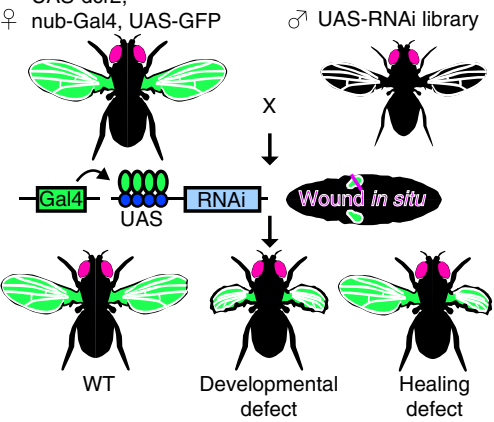

h

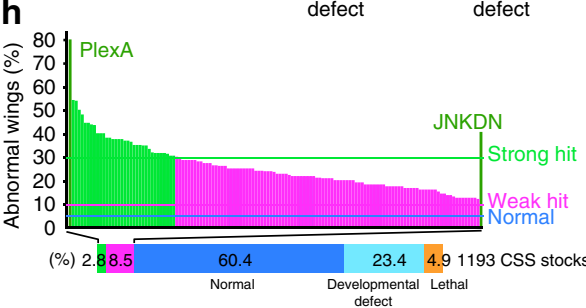

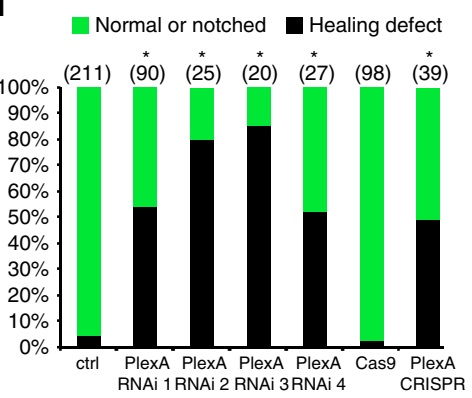

j
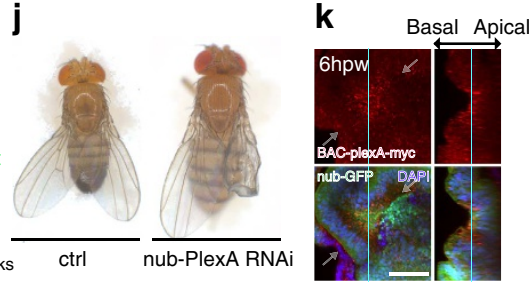

Figure 1 | An RNAi screen identifies a role for PlexA in wing disc repair. (a) Diagram of in situ wounding of wing discs (Supplementary Movie 1). (b) Damage induces a transient calcium flash in wing discs (Supplementary Movie 2). The top inset is a kymograph derived from the region shown in the rectangular box. (c,d) F-actin accumulates and the AP-1 reporter is activated at the wound edges (white arrows), which have fused by $6 \mathrm{~h}$ post wounding (h.p.w.). (e,f) Adult wing phenotypes after wounding wing discs of $L 3$ larvae. (g,h) Scheme of the wing disc-specific RNAi screen and classes of phenotypes obtained. Overall, 1,193 CSS RNAi lines were used. (i,j) Four independent PlexA RNAi transgenes and the wing disc-specific PlexA knockout with CRISPR induce healing defects. ${ }^{\star} P<0.05$, two-tailed $\chi^{2}$-test with the Bonferroni correction (RNAi), two-tailed $\chi^{2}$-test (CRISPR). (k) PlexA expression in wounded wing discs of PlexA-myc BAC transgenic flies. Arrows indicate the wound location. Scale bars, $50 \mu \mathrm{m}$. 
pouch. Under these conditions, as with RNAi lines that targeted PlexA, proper repair was not observed (Fig. 1i and Supplementary Fig. 1c). PlexA is indeed expressed in wing discs, and its expression was more pronounced around wound edges (Fig. 1k and Supplementary Fig. 1d-f).

PlexA regulates wound closure in the epithelium of the notum. In order to directly visualize the effect of PlexA inhibition on the dynamics of wound closure, we examined the closure of wounds generated by a laser in the epithelium of the pupal notum, which is amenable to live imaging. In control pupae, laser-generated wounds closed within $120 \mathrm{~min}$ (Fig. 2a and Supplementary Movie 3). In contrast, expression of a PlexA RNAi resulted in a delay in wound closure, taking 180-240 $\mathrm{min}$. In the pupal notum, it was also evident that the PlexA RNAi made the cell boundaries, which were visualized using E-cadherin-GFP, more wiggly in the region around the wound (Fig. 2b,c). To better analyse this phenomenon, we measured the ratio between the total junction length and the distance between the correspondent vertexes for each condition (Fig. 2d). This ratio fluctuates along time in both control and PlexA RNAi (Fig. 2e,f), but in the latter there is a peak of the ratio $\sim 5 \mathrm{~min}$ post wounding, when the changes in cell outlines are more pronounced. Using this time point as a post-wounding reference we measured this ratio using cell outlines from five independent pupae for each condition, both before and after wounding (Fig. 2g). With this analysis we observed that even before wounding, PlexA inhibition is sufficient to make the junction morphology more wiggly when compared with control, but also that when the tissue is challenged by a laser wound this phenotype is significantly more pronounced. Wiggly cell outlines have been previously observed when cell-cell adhesion is altered $^{17,18}$. Thus, in the epithelium of the notum, PlexA function is necessary for efficient wound closure and a reduction in PlexA function likely results in alterations in cell adhesion.

PlexA signalling regulates wound repair. The role of plexins has been best characterized in the context of axonal pathfinding ${ }^{19-22}$. Plexins are transmembrane proteins that bind to their ligands, semaphorins, and then regulate the actin cytoskeleton to mediate neurite repulsion ${ }^{19-21,23}$. The flavoprotein oxidase Mical has been shown to function downstream of plexins in this process. To determine whether these other components of the semaphorin/ plexin pathway are also involved in tissue repair in imaginal discs, we first tested whether they were expressed in imaginal discs. Expression of all the known plexins, semaphorins and Mical was detected in discs by reverse transcriptase PCR (RT-PCR; Supplementary Fig. $2 \mathrm{a}-\mathrm{c})$. We then tested the requirement of Semala and Semalb, which are ligands for PlexA ${ }^{19-22}$, in the repair of damaged discs. While knockdown of either Sema1a or Sema1b had little effect (Supplementary Fig. 2d), simultaneous knockdown of both Semala and Semalb increased the frequency of adult wings of abnormal morphology on the wounded side (Fig. 3a,b). The weaker effect of knockdown of Semala and Sema1b when compared with PlexA could be because of either partial knockdown or redundancy with other semaphorins. Overexpression of a truncated form of PlexA that lacks a a

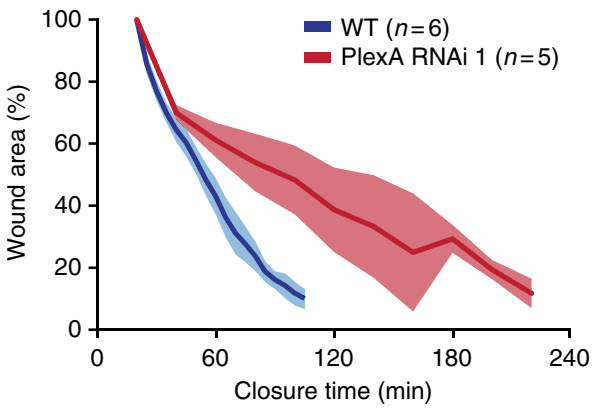

d



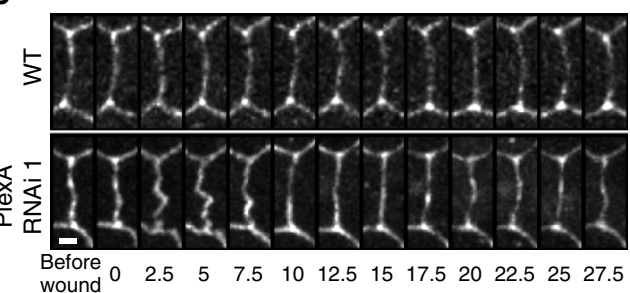

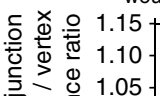
5
0 -
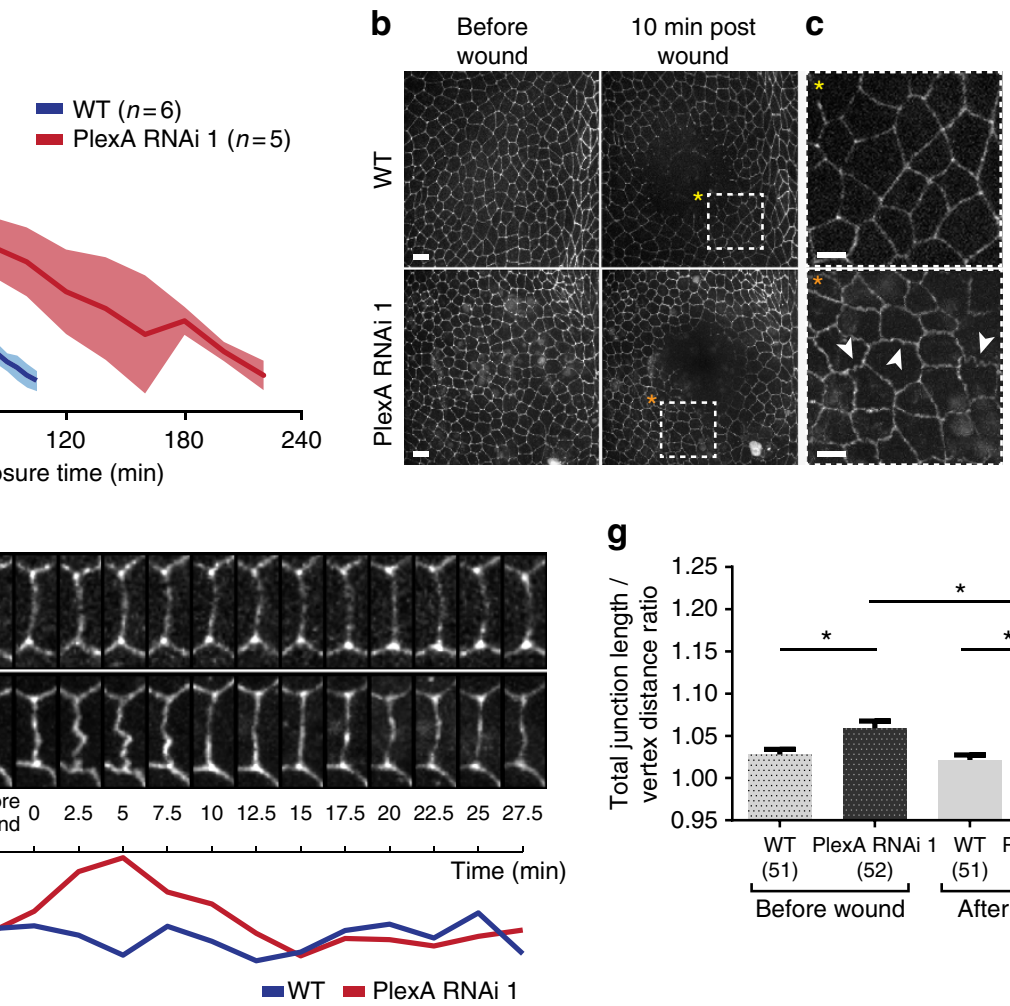

Figure 2 | PlexA regulates wound closure in the epithelium of the notum. (a) PlexA RNAi slows down wound closure upon laser wounding in the pupal notum at 13 APF. Shadowed areas represent s.d. (b,c) Expression of E-Cad-GFP in notum epithelia. Cells in the PlexA RNAi epithelium exhibit wiggly cell boundaries around the wound (indicated with white arrowheads) when compared with controls. Scale bars, (b) $10 \mu \mathrm{m}$ and (c) $5 \mu \mathrm{m}$. (d) Schematic diagram of the ratio used to quantify the membrane defects. (e,f) Quantification of the junction length and vertex distance ratio over time in controls and PlexA RNAi. The membrane morphology change reaches its maximum at 5 min after wounding. Scale bar, $2 \mu \mathrm{m}$. ( $(\mathbf{g})$ Junction length and vertex distance ratio for controls and PlexA RNAi both before and after wound for five independent pupae for each condition. Error bars represent s.e.m. ${ }^{\star} P<0.05$; Kruskal-Wallis test with Dunn's post-test. 

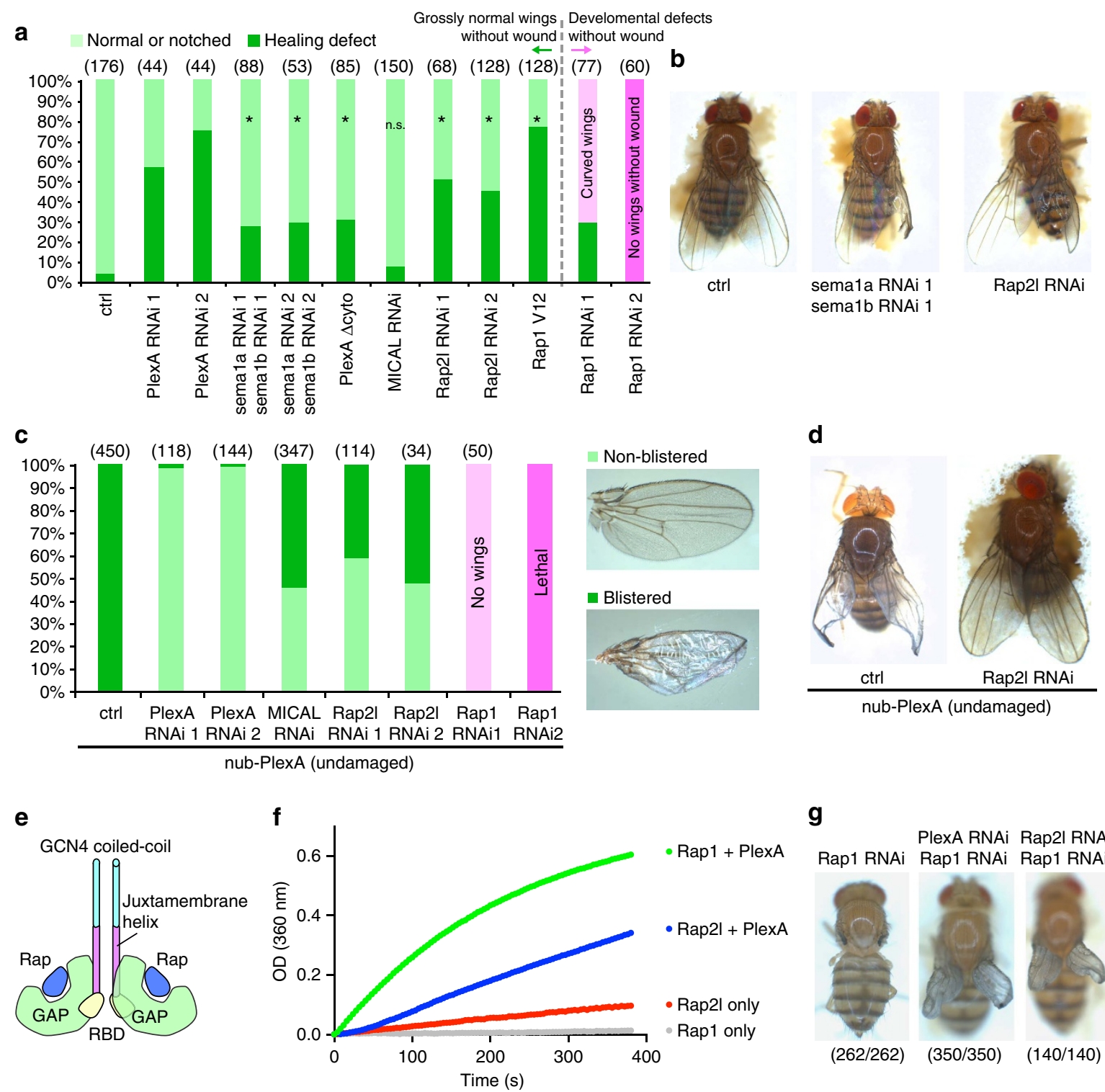

g

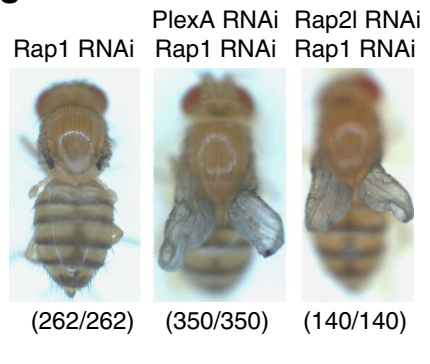

Figure 3 | PlexA signaling regulates wound repair. $(\mathbf{a}, \mathbf{b})$ Double knockdown of sema1a and sema1b, expression of PlexA $\Delta$ cyto, knockdown of Rap2l or expression of constitutively active Rap1 V12 perturbs wound healing but MICAL knockdown does not. Note that only right wings are wounded. The flies were held in a hole of a sponge, which looks brown beneath flies, for image acquisition. ${ }^{\star} P<0.05$, two-tailed $\chi^{2}$-test with the Bonferroni correction.

$(\mathbf{c}, \mathbf{d})$ Ectopic expression of PlexA in the wing pouch induces blisters in adult wings. This effect is prevented by Rap2l or MICAL knockdown and enhanced by

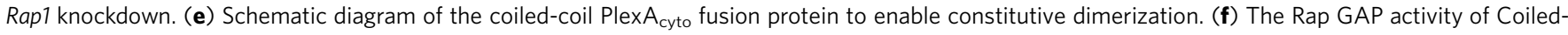
coil PlexA $A_{\text {cyto }}$ was measured using the photometric assay. The slope of the linear portion of the reaction curves reflects the initial rate of GTP hydrolysis (see Methods for details). The rate of GTP hydrolysis of Rap1 catalysed by PlexA (0.166 O.D. per min) is approximately ninefold higher than that of Rap2I (0.019 O.D. per min). (g) Rap1 knockdown-induced loss of adult wings is suppressed by inhibition of PlexA or Rap2l.

cytoplasmic domain (PlexA $\Delta$ cyto) ${ }^{23}$ also resulted in wing abnormalities following wounding (Fig. 3a). This indicates that PlexA $\Delta$ cyto likely functions in a dominant-negative capacity by competing for the ligands of endogenous PlexA and that PlexAmediated intracellular signalling is likely important for tissue repair. Interestingly, Mical inhibition did not cause a significant disruption of adult wing morphology following wounding, even though Mical knockdown by RNAi was efficient (Fig. 3a and Supplementary Fig. 2c). Taken together, these findings suggest that PlexA functions together with Semala and Semalb in tissue repair.

Vertebrate plexins function as a GTPase-activating protein (GAP) for the small GTPase Rap, thereby reducing the levels of active (GTP-bound) Rap. Semaphorin binding is predicted to increase the GAP activity of plexins through their dimerization, thereby reducing Rap activity ${ }^{24,25}$. In Drosophila, mutations in the Rap1 gene disrupt morphogenesis in the embryo ${ }^{26}$. Rap1 regulates the stability of adherens junction ${ }^{27}$ and decreasing the levels of Rap-GTP by expression of a GAP, Rapgap1, has been shown to increase the mobility of adherens jnctions by reducing their coupling to the actin cytoskeleton ${ }^{28}$. Thus, an attractive hypothesis is that the activation of PlexA signalling in damaged tissue increases GAP activity for Rap1 and thus allows cell rearrangements and tissue remodelling.

Drosophila has two rap-family GTPases, Rap1 and Rap21, which are orthologues of vertebrate Rap1 and Rap2, respectively. In at least some situations, such as endothelial cell adhesion in mammals, Rap1 and Rap2 function antagonistically ${ }^{29}$. Moreover, 
mammalian plexins have a higher GAP activity for Rap1 (ref. 24). We therefore examined the effect of reducing the levels of both Rap1 and Rap2l. Reducing the levels of Rap1 caused developmental defects even in the absence of injury (Fig. 3a), consistent with its role in embryonic morphogenesis and adherens junction formation ${ }^{26,27}$. In contrast, inhibition of Rap2l caused injury-dependent phenotypic abnormalities that were similar to those elicited by reducing PlexA levels (Fig. 3a,b). This is consistent with the possibility that both Rap2l and PlexA antagonize Rap1 function.

To further characterize the relationship between PlexA and the rap genes, we manipulated activity of the rap GTPases under conditions of PlexA overexpression. When PlexA was overexpressed, the adult wings showed blistering (Fig. 3c,d), suggesting a lack of proper adhesion between the cells on the dorsal and ventral surfaces. The blistering phenotype caused by PlexA overexpression was suppressed efficiently by knockdown of either Rap2l or Mical (Fig. 3c,d). In contrast, reducing Rap1 levels while overexpressing PlexA resulted either in a complete absence of wings or in lethality (Fig. 3c). Since the blistering phenotype elicited by overexpression of PlexA alone can be changed to phenotypes of no wings or lethality simply by increasing expression levels (Supplementary Fig. 2e), we conclude that Rap1 reduction likely enhances the effect of PlexA overexpression. If, as in mammals, PlexA reduces the activity of the rap GTPases in Drosophila, reducing Rap levels should enhance the PlexA overexpression phenotype. Indeed, we observed this kind of interaction with Rap1 but not with Rap2I. Thus, our genetic data are consistent with PlexA functioning primarily as a GAP for Rap1 in Drosophila tissues rather than for Rap2l.

To test whether Drosophila PlexA possesses bona fide GAP activity for Rap GTPases, we performed an in vitro GAP assay. We purified the cytoplasmic domain of Drosophila PlexA connected to the GCN4 coiled-coil domain to promote its dimerization and performed a photometric GAP assay using purified Drosophila Rap1 and Rap2l as substrates, as previously described ${ }^{24}$. We found that PlexA catalyses GTP hydrolysis of rap GTPases, possessing about ninefold higher activity towards Rap1 compared with Rap2l (Fig. 3e,f), which is consistent with the predictions made by the genetic interactions.

As mentioned above, mammalian Rap1 and Rap2 have antagonistic roles in the context of endothelial cell adhesion; Rap1 promotes adhesion while Rap2 reduces adhesion ${ }^{29}$. Since knockdown of either PlexA or Rap2l, each of which can antagonize Rap1, impairs wound healing, the healing defect might be caused by a failure to downregulate Rap1 activity. Consist with this possibility, expression of a constitutively active Rap1 (V12) impaired wound repair (Fig. 3a). Further, the wingdefect phenotype caused by Rap1 knockdown was similarly suppressed by inhibition of PlexA or Rap2l, which would be predicted to restore Rap1 activity to some extent (Fig. 3g). Thus, taking our genetic and biochemical data together, we propose that PlexA regulates epithelial wound repair by functioning as a GAP primarily for Rap1. Since PlexA demonstrates GAP activities towards both Rap1 and Rap2l but with a higher activity for Rap1 in vitro, Rap1 could potentially suppress Rap2l regulation by PlexA in cells. Although Mical knockdown suppresses the PlexA overexpression phenotype, the absence of a healing defect with Mical knockdown might imply that Mical functions as an effector for PlexA only in a subset of functions or that the function of Mical is redundant in some contexts. Indeed, C. elegans does not have a recognizable Mical orthologue despite a clear role for semaphorin-plexin signalling in axonal pathfinding ${ }^{30}$.
PlexA regulates delamination of epithelial cells. To examine the role of PlexA in imaginal discs, we first generated PlexA ${ }^{R N A i_{-}}$ expressing clones in undamaged discs. These clones were indistinguishable from control clones, indicating that mutant cells proliferate normally (Supplementary Fig. 3a,b). When we compared damaged discs expressing PlexA ${ }^{R N A i}$ with wild-type discs, the initial fusion of the two fragments, F-actin accumulation at the wound edge and JNK activation were indistinguishable from wounded wild-type discs (Supplementary Fig. 3c-e). In contrast, inhibition of JNK function interferes with these early events in wound healing ${ }^{15}$. However, unlike wild-type discs, healing PlexA $A^{R N A i}$ discs had a large cleft when visualized from the side of the peripodial epithelium (Fig. 4a,b and Supplementary Movie 4). In addition, wild-type discs had many small-volume cells with intense GFP fluorescence that resembled apoptotic cells basal to the epithelial layer. In $n u b>P l e x A^{R N A i}$ discs some of these cells had been basally extruded, while others were observed throughout the thickness of the epithelium (Fig. 4c-e). These cells contained activated caspase, indicating that they were undergoing apoptosis (Fig. 4f). Thus, PlexA function appears necessary for efficient extrusion of dying cells.

To test whether increasing PlexA activity is sufficient to promote cell extrusion, we overexpressed PlexA in undamaged discs and observed extensive basal extrusion of cells (Fig. 4g). Most of these cells did not express activated caspase (Supplementary Fig. 3f,g), indicating either that PlexA promotes the extrusion of live cells or that the levels of caspase activation are below the threshold of detection by this method. To test whether PlexA functions in the cells being extruded or in their neighbours, we expressed PlexA in scattered single cells. PlexA-overexpressing cells were preferentially dislocated towards the basal side of the epithelium (Fig. 4j-l), indicating a cellautonomous role of PlexA in this process. Dislocation of cells to the basal side by ectopic PlexA expression was enhanced by Rap1 inhibition and suppressed by Rap1 activation, consistent with the notion that PlexA promotes cell extrusion by reducing Rap1 activity (Fig. 4h,i,m). Furthermore, blocking apoptosis in damaged discs by expression of the baculoviral anti-apoptotic protein, $\mathrm{p} 35$, resulted in an increased number of adult wings with morphological defects upon wounding (Supplementary Fig. 3h,i). Thus, the death of damaged cells, as well as their efficient extrusion basally, might be a prerequisite for efficient repair of damaged epithelia with PlexA having a role in cell extrusion. This is consistent with the reported necessity for apoptotic cell death in damaged tissue before efficient regeneration in Hydra and in tadpole tails ${ }^{31,32}$

To further explore the role of PlexA in basal extrusion of cells, we examined its requirement in a different situation. Inhibition of Rho kinase (Rok) results in misorientation of mitotic spindles causing cells to delaminate basally from the epithelium and subsequently undergo apoptosis ${ }^{33}$. In these discs, a condensation of F-actin is seen in the basal portion of the epithelium that separates the epithelium from the basally extruded cells (Fig. 4n). Concurrent expression of PlexA $A^{R N A i}$ does not prevent either apoptosis or the basal displacement of these cells, but it results in the retention of the delaminating cells within the basal portion of the epithelium rather than in their extrusion (Fig. 4o). This indicates that PlexA is also important for the epithelial remodelling induced by Rok inhibition.

PlexA mediates uncoupling of F-actin and adherens junctions. How does PlexA dislocate cells to the basal side of the epithelial layer? In addition to the continuous F-actin accumulation along the wound edge, we also observed sporadic foci of F-actin accumulation in the vicinity of the wound (Fig. 5a), especially 

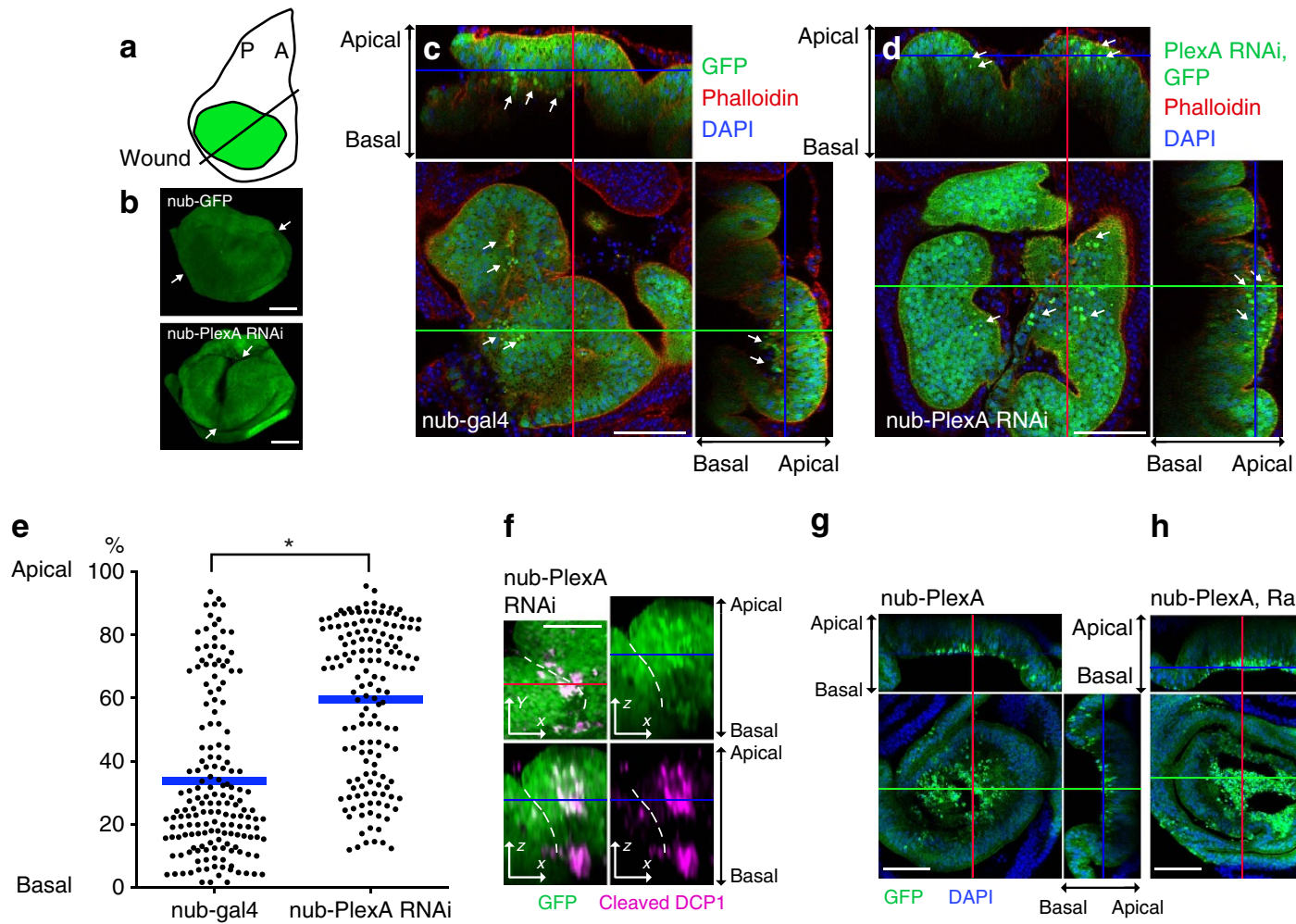

f

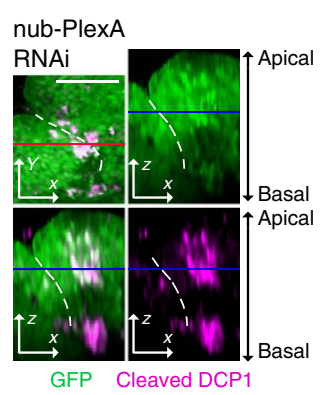

g

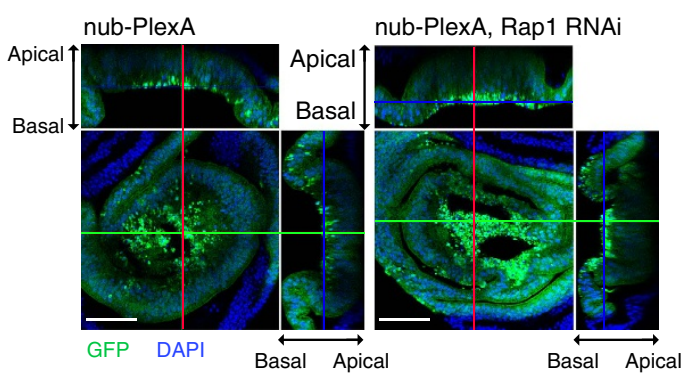

j

\section{i}

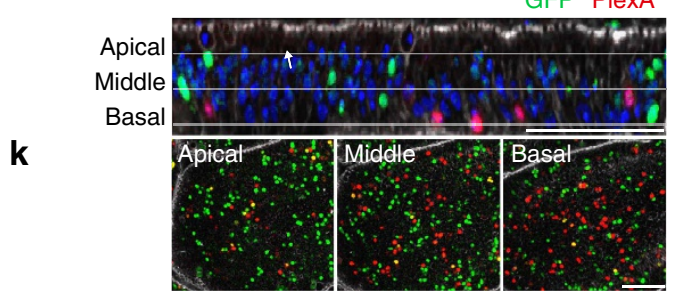

I

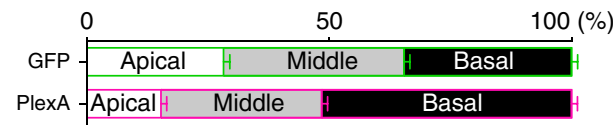

m

\begin{tabular}{|c|c|c|c|c|}
\hline & & & 50 & \\
\hline Control & Apical & H & Middle & Basal \\
\hline PlexA & Apical H & Middle & -1 & \\
\hline Control & Apical & $\mathrm{H}$ & Middle & Basal \\
\hline rap1 v12 & Apical & $\mathrm{H}$ & Middle & Basa \\
\hline
\end{tabular}

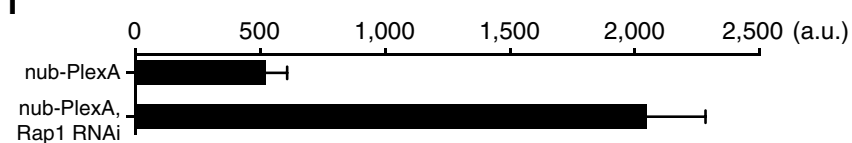

n

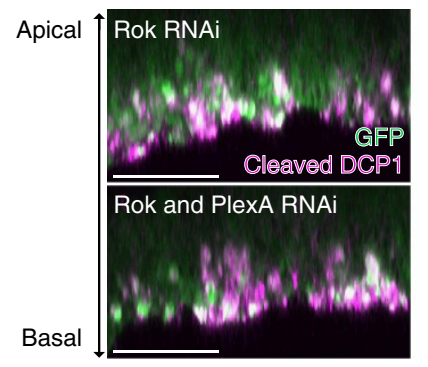

o



Figure 4 | PlexA regulates delamination of epithelial cells. (a) Diagram of a bisected wing disc ( $A$, anterior; P, posterior). (b) 3D reconstruction of wounded wing discs at 18 h.p.w. (Supplementary Movie 4). White arrows indicate the wound sites. (c,d) Control discs have many basally extruded cells at18 h.p.w. In PlexA RNAi discs, unextruded round cells with intense GFP signals are observed in the middle of the epithelial layer at 18 h.p.w. White arrows indicate examples of round cells with intense GFP fluorescence. (e) $z$ axis location of the round cells with intense GFP fluorescence in damaged wing discs at 18 h.p.w. ${ }^{\star} P<0.05$; two-tailed Mann-Whitney U-test. (f) The small cells with intense GFP signals in the middle of the epithelial layer contain the activated effector caspase DCP-1. Dotted lines indicate the wound edge. ( $\mathbf{g}, \mathbf{h})$ Ectopic expression of PlexA induces basal cell extrusion, which is enhanced by concomitant inhibition of Rap1. (i) Quantification of basally extruded cells. Error bars represent s.e.m. (nub-PlexA: $n=6$, nub-PlexA, Rap1 RNAi: $n=6$ ). (j-I) PlexA expression in single-cell clones. Cells expressing PlexA also express RFP. Control cells express GFP alone. PlexA expression displaces cells to the basal side cell autonomously. Error bars represent s.e.m. $(n=17$ discs). ( $\mathbf{m})$ Basal displacement of cells by PlexA expression in single-cell clones is suppressed by Rap1 activation. Error bars represent s.e.m. (top: $n=5$, bottom: $n=6$ ). (n) Rok RNAi-mediated apoptosis is not affected by PlexA inhibition. (o) Rok RNAi induces cell delamination, with F-actin separating the epithelium from the basally extruded cells. PlexA inhibition perturbs Rok RNAi-induced cell delamination. White arrows indicate the plane of F-actin accumulation. Scale bars, $50 \mu \mathrm{m}$.

around the dying cells, which are characterized by intense GFP fluorescence as mentioned previously (Fig. $4 \mathrm{f}$ ). At higher magnification, each of the F-actin foci could be seen as a ring structure surrounding the cell (Fig. 5b). Such actin rings are typically observed around cells undergoing apoptosis and extrusion $^{34}$. Upon PlexA inhibition, the F-actin accumulation associated with dying cells was significantly reduced (Fig. 5a). Conversely, ectopic expression of PlexA in the whole-wing disc 
a
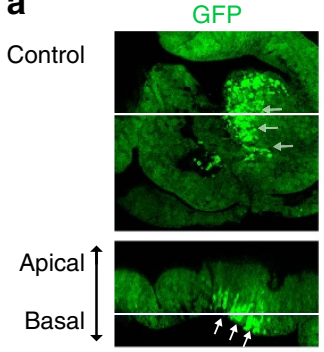

PlexA

RNAi

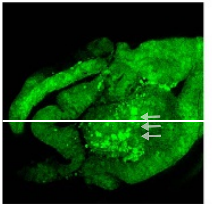

Apical

Basal

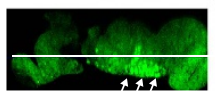

Phalloidin
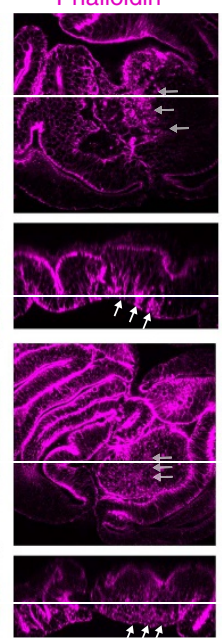
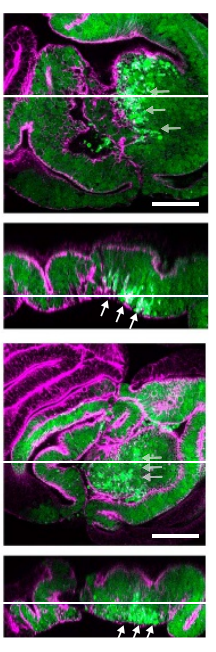

6hpw b

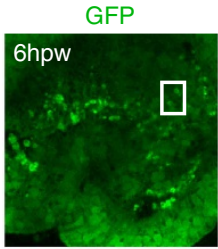

F-actin
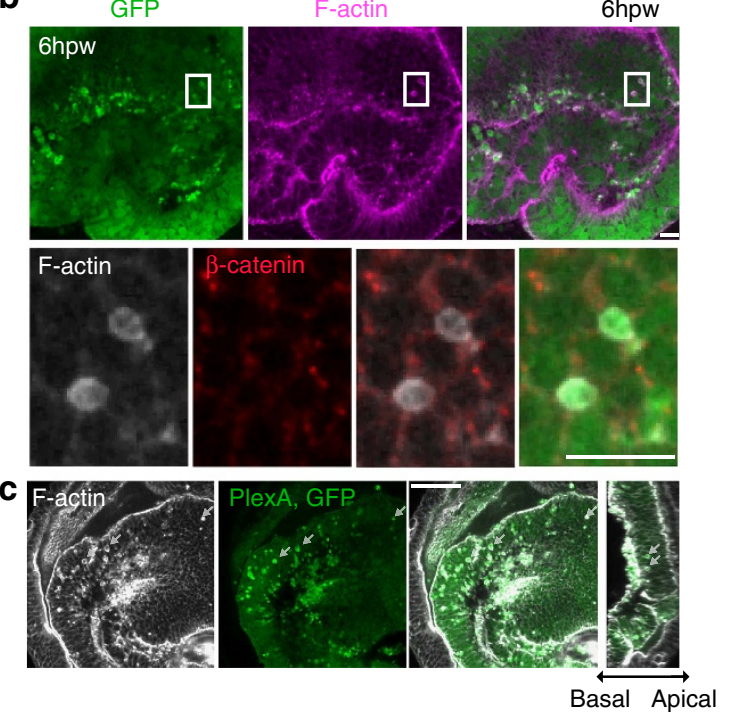

d

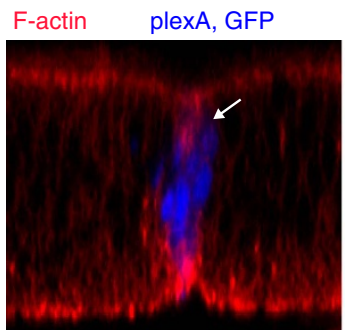

$\beta$-catenin

plexA, GFP
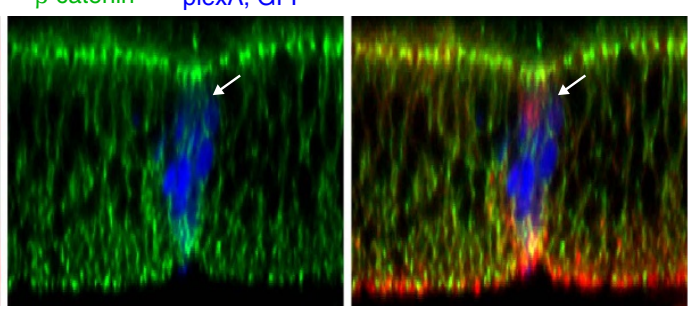

Ratio (F-actin/ $\beta$-catenin) plexA, GFP
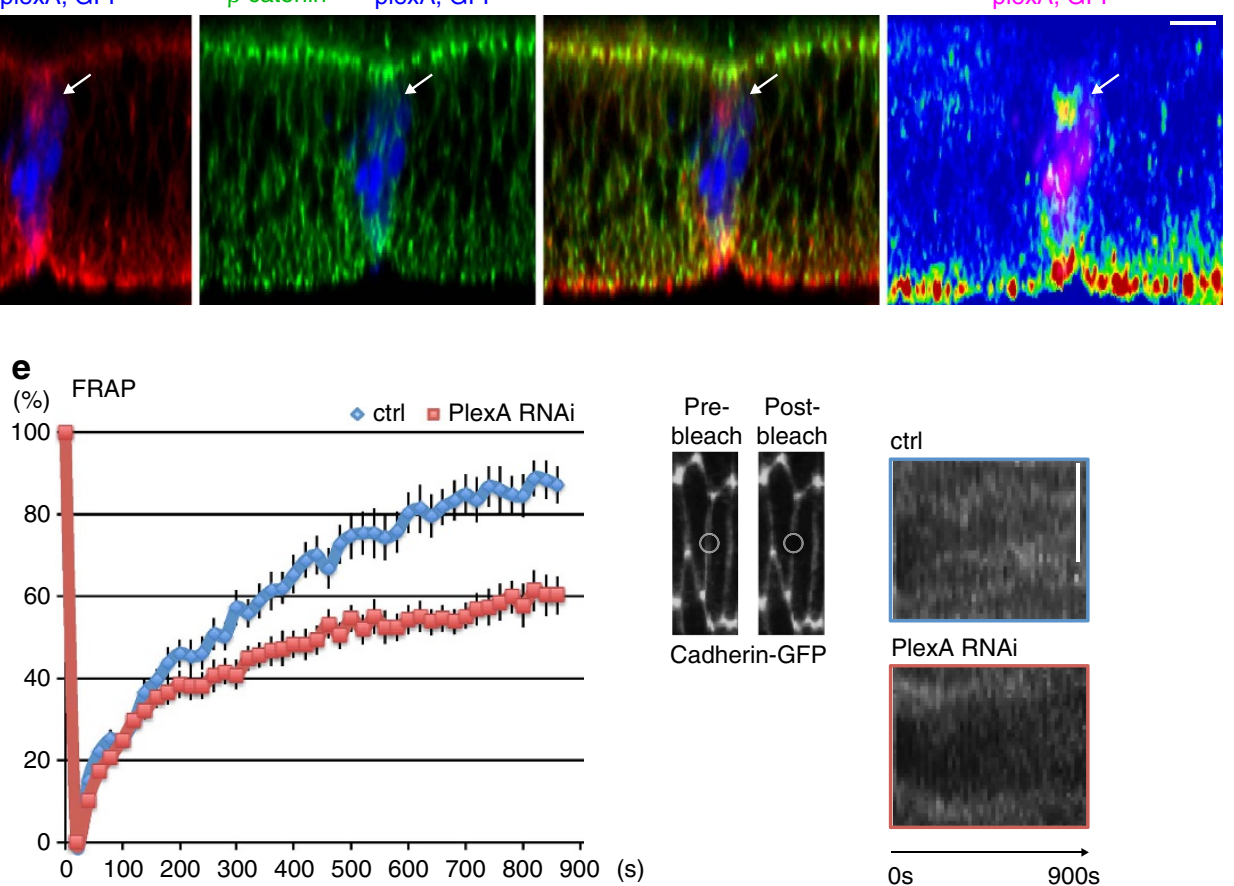

Pre- Post-

bleach bleach
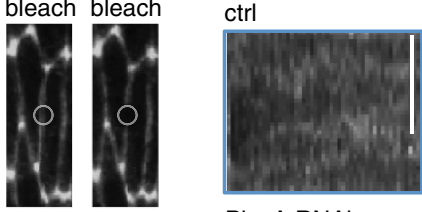

Cadherin-GFP

PlexA RNAi



$\overrightarrow{\text { os } \quad 900 \mathrm{~s}}$

Figure 5 | PlexA mediates uncoupling of F-actin and adherens junctions. (a) In control discs the round cells with intense GFP signals have pronounced accumulation of F-actin at 6 h.p.w. In PlexA RNAi discs, these cells have less accumulation of F-actin. White arrows indicate examples of round cells with intense GFP fluorescence. Data are representative of more than 10 images. (b) The round cells with intense GFP signals have actin rings around them at 6 h.p.w. (c) Extruded cells by ectopic expression of PlexA have actin rings around them (indicated with white arrows). Data are representative of more than five images. (d) Ratiometric analysis shows that cells with elevated PlexA have more F-actin uncoupled with adherens junctions compared with the surrounding cells. (e) FRAP analysis of cadherin-GFP in the pupal notum at 13 APF. PlexA RNAi inhibits fluorescence recovery of cadherin-GFP after photobleaching compared with control. Error bars represent s.e.m. (ctrl: $n=20$, PlexA RNAi: $n=18$ ). Right pictures show the process of photobleaching and kymographic analyses. Scale bars, $50 \mu \mathrm{m}(\mathbf{a}, \mathbf{c}), 10 \mu \mathrm{m}(\mathbf{b}, \mathbf{d})$ and $5 \mu \mathrm{m}(\mathbf{e})$.

gave rise to many extruded cells with actin rings (Fig. 5c). Further, ectopic expression of PlexA in multiple-cell or single-cell clones for a limited time also induced F-actin accumulation around cells, especially on their apical side (Fig. 5d and Supplementary Fig. 4a,b). Importantly, while F-actin accumulates in or immediately around cells that are being extruding following wounding or PlexA overexpression, the polymerized F-actin does not induce a concomitant accumulation of adherens junctions, which was analyzed by measuring the ratio of F-actin to $\beta$-catenin (Fig. 5b,d and Supplementary Fig. 4a). Since Rap1 strengthens the coupling of F-actin and adherens junctions $28,29,35,36$, the lack of coupled accumulation of these two proteins is suggestive of reduced Rap1 activity resulting from the ability of PlexA to function as a GAP for Rap1.

The finding that ectopic PlexA expression induces an uncoupling of F-actin and adherens junctions prompted us to 
investigate the role of endogenous PlexA in the dynamic remodelling of adherens junctions because Rap1-mediated coupling of F-actin and adherens junctions is thought to stabilize adherens junctions ${ }^{28,29,35,36}$. Since it was difficult to perform a detailed analysis of junction dynamics in the wounded wing disc, we once again used the pupal notum epithelium to visualize adherens junction dynamics. PlexA inhibition affects not only wound repair (Fig. 2a) in the notum but also its normal development. Reducing PlexA levels in the notum results in an increased width of the midline region separating the microchaetes from $\sim 10$ to $20 \mu \mathrm{m}$ (Supplementary Fig. 5a). This is the region where the two wing discs fuse with each other during normal pupal development, a process that bears at least some superficial similarities to the rejoining of fragmented discs. The analysis of the pupal notum showed that PlexA inhibition induces a cleft in the midline (Supplementary Fig. 5b), which is reminiscent of the healing defect of the damaged wing disc (Fig. 4b). Although cell extrusion is known to occur in the midline of the notum during development ${ }^{37,38}$, we did not see an obvious effect of PlexA RNAi in reducing cell extrusion (Supplementary Fig. 5c), suggesting that the extent to which PlexA mediates cell extrusion depends on its context. To examine the dynamics of adherens junctions, we performed a fluorescence recovery after photobleaching (FRAP) analysis of cadherin-GFP in the epithelium of the midline notum. PlexA RNAi slowed down FRAP of cadherin-GFP (Fig. 5e), indicating that cadherin is less dynamic and more stable in the PlexA RNAi epithelia. This suggests that PlexA functions to reduce the stability of adherens junctions, thereby allowing the movement of cells in the epithelium with respect to their neighbours. Such cellular movements may be necessary for the extrusion of damaged cells and the subsequent remodelling of the epithelium to enable the re-establishment of epithelial continuity following damage.

Plexin A1 regulates wound repair in zebrafish fins. Finally, to test whether plexins have a role in repairing damaged tissue in vertebrates, we utilized the well-characterized system of tail fin regeneration in zebrafish larvae, where defects in the earliest stages of wound repair impair fin regeneration at much later stages $^{2}$. The zebrafish, Danio rerio, has four PlexA orthologues, plexin $A 1, A 2, A 3$ and $A 4$. plexin $A 1$ and $A 3$ are expressed highly in larval tail fins (Fig. 6a). Reducing the levels of Plexin A3 using antisense mopholino oligonucleotides (MO) did not affect regeneration (Supplementary Fig. 6a-d). Reducing levels of Plexin A1 caused defects in early embryonic development. However, by using photo-morpholinos, which can be activated by ultraviolet light, we reduced Plexin A1 function 1 day post fertilization without significantly affecting normal development (Fig. 6b,c). Under these conditions, regeneration of the amputated tail fin was impaired, thus demonstrating a role for a PlexA orthologue in tissue repair in vertebrates (Fig. 6d-f). In addition to eliciting phenotypes using MOs, we also found that Plexin A1 inhibition through injection of Cas9 mRNA and two different gRNAs for plexin A1 also impaired tissue repair (Fig. 6g).

We also examined earlier stages in wound healing by live imaging. During wound repair in tail fins, we observed epithelial cell extrusion around the wound edge (Fig. 6h and Supplementary Movie 5). Extruded cells had features of cells undergoing apoptosis (Fig. 6i). As wound healing proceeded, the number of apoptotic cells around wounds decreased in wild-type tail fins (Fig. 6j,k) but less so when the levels of Plexin A1 were reduced. Thus, consistent with our observations in Drosophila, reduction of Plexin A1 inhibited removal of the apoptotic cells around wounds in zebrafish. Although we have not defined the precise mechanism by which Plexin A1 regulates wound healing in zebrafish, these observations may reflect similar functions for plexins in wound repair in Drosophila and zebrafish.

\section{Discussion}

We have described a role for Drosophila PlexA and for its zebrafish orthologue Plexin A1 in wound repair. PlexA exerts its function as a GAP for Rap1, which promotes coupling of F-actin and adherens junctions and thereby stabilizes adherens junctions $28,29,35,36$. On the basis of our data in Drosophila, we propose the following mechanism by which PlexA regulates epithelial wound repair (Supplementary Fig. 7a,b). Injury upregulates PlexA in cells in the vicinity of the wound (Fig. 1k), which leads to inhibition of Rap1 (Fig. 3f). PlexA-mediated Rap1 inhibition uncouples F-actin and adherens junctions, thus facilitating junctional remodelling (Figs 2 and 5). This is necessary for proper cell rearrangements including cell extrusion (Fig. 4), and the subsequent epithelial remodelling that occurs during wound repair. Since an actin regulator Mical functions downstream of PlexA in a context-dependent manner ${ }^{23}$ (Fig. 3c), it is possible that PlexA could also influence wound repair and epithelial remodelling through an additional mechanism such as Micalmediated regulation of F-actin.

A recent study reported that PlexinB and its ligands Sema4b, $4 \mathrm{~d}$ and $4 \mathrm{~g}$ regulate renal wound repair and mitotoic spindle orientation in mammals ${ }^{39}$. We did not observe an obvious effect of PlexA inhibition either on mitotic orientation or mitosis in Drosophila. However, given that the experimental settings and the types of plexins and semaphorins differ between the two studies, it is possible that the other plexin and semaphorins have additional functions in mediating repair of Drosophila epithelia.

Finally, our findings are worth examining from an evolutionary perspective. Although we do not know whether the role of Plexin A1 in wound repair in zebrafish is mechanistically the same as that in Drosophila imaginal discs, our demonstration of a role for plexins in epithelial wound repair in metazoans that belong to two different superphyla (Ecdysozoa and Deuterostomia) suggests that this function could be evolutionarily ancient (Supplementary Fig. 7c). Plexins and semaphorins exist in all eumetazoa, in the placozoan Trichoplax adhaerens and in the sponge Amphimedon queenslandica. Thus, their origin predates the evolution of the nervous system. If the ancestral role for plexins is in wound repair, possibly in remodelling of adherens junctions and extruding damaged epithelial cells, it may have been subsequently modified in the nervous system to function in neurite repulsion. We therefore suggest that some of the other genes that have been studied primarily in the nervous system may also have a hitherto unsuspected role in epithelial wound repair.

\section{Methods}

Drosophila stocks. Flies were crossed and raised at $25^{\circ} \mathrm{C}$ unless otherwise noted. For wild-type controls, Oregon-R (Bloomington stock centre (BL) 4269) was used unless otherwise specified. For the primary screening of CSS genes, RNAi stocks were collected from the BL and the Vienna Drosophila RNAi center (VDRC) based on a previous report ${ }^{16}$ and the LOCATE database (http://locate.imb.uq.edu.au).

Several RNAis were also obtained from the National Institute of Genetics (NIG).

The following fly stocks were used in this study:

UAS-dcr2; nub-gal4 (BL25754)

UAS-GFP

UAS-his2A::RFP (a gift from Dr Knoblich)

UAS-GCaMP3 (BL32116)

UAS-PlexA RNAi 1 (BL30483)

UAS-PlexA RNAi 2 (VDRC4740)

UAS-PlexA RNAi 3 (VDRC27240)

UAS-PlexA RNAi 4 (VDRC107004)

UAS-bsk DN (JNK DN)/TM6b

UAS-MICAL RNAi (BL31148)

UAS-Rap2l RNAi 1 (BL51840)

UAS-Rap2l RNAi 2 (VDRC107745)

UAS-Rap1 RNAi1 (BL29434)
} 
a

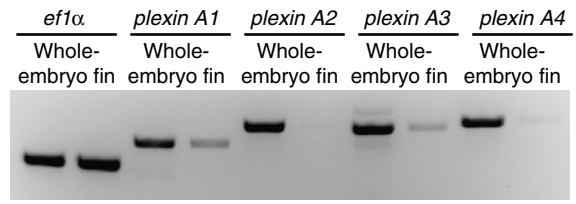

b

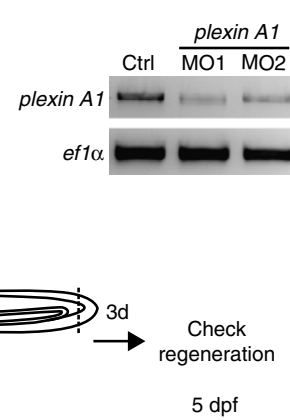

d

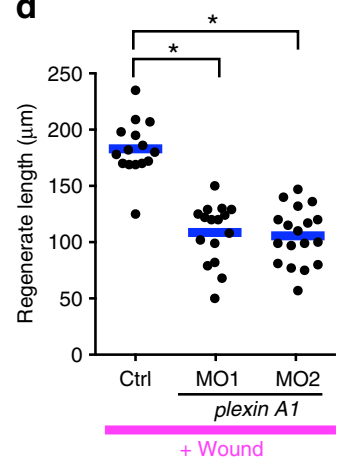

e

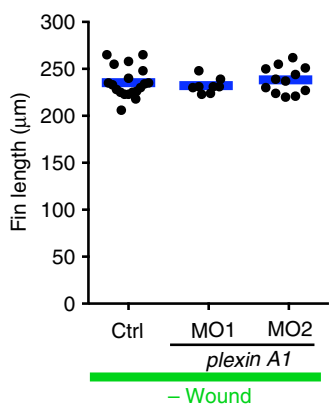

f

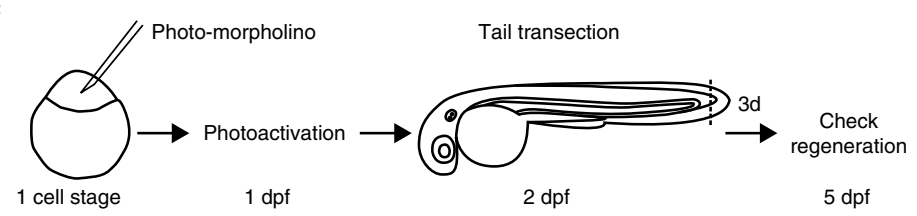

Control
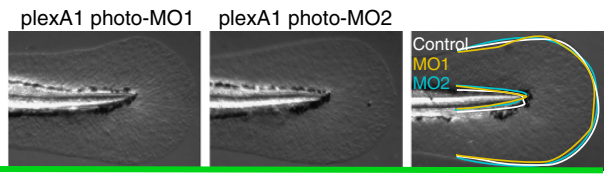

g
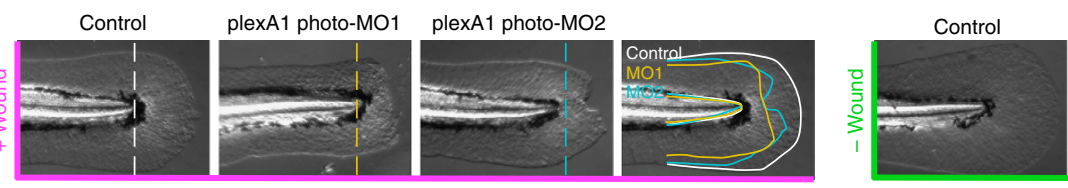

j

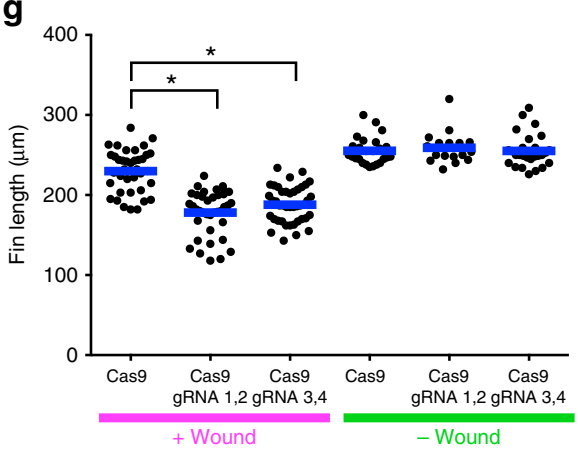

i

h
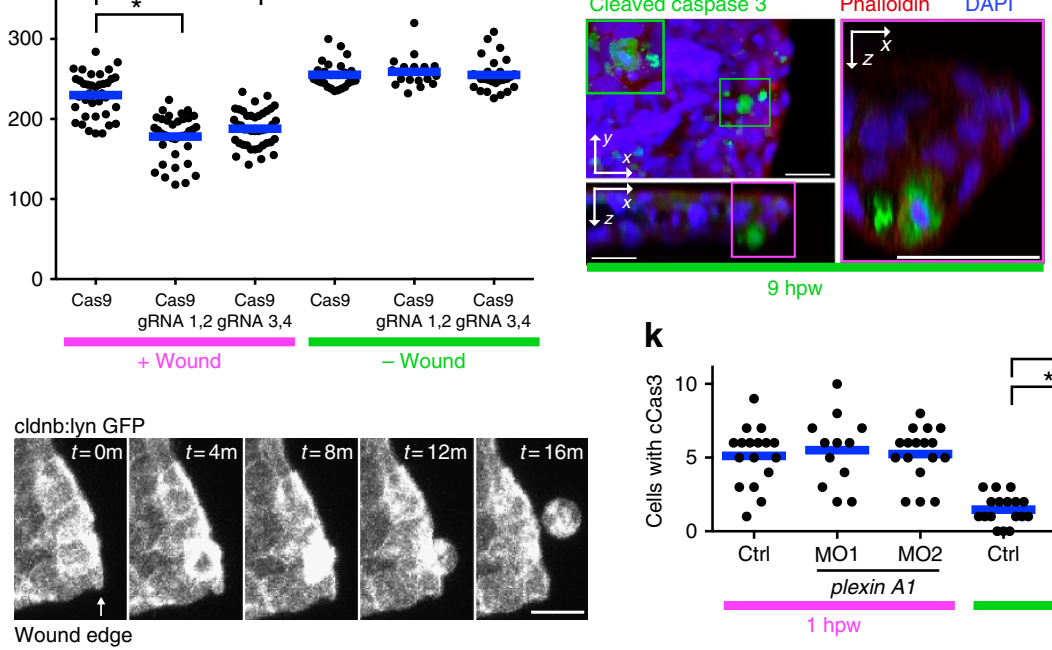

DAPI


k

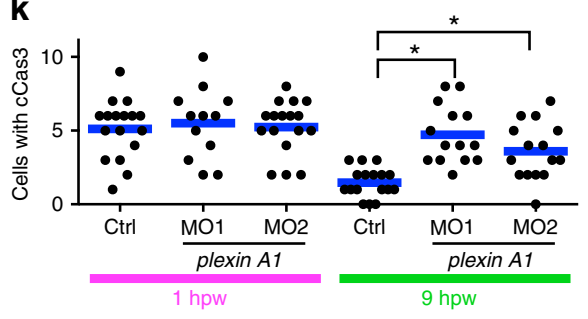

Figure 6 | Plexin A1 regulates wound repair in zebrafish fins. (a) RT-PCR of plexins. plexin A1 and A3 are relatively enriched in tail fins at 2 d.p.f. (b) RT-PCR of plexin A1 following knockdown. Two independent splice morpholinos were used. (c) Diagram of photo-morpholino injection, photoactivation and tail transection. (d) Regenerated fin length at 3 days post wounding (d.p.w.), i.e., 5 d.p.f. ${ }^{\star} P<0.05$; one-way ANOVA with Dunnett's post-test. (e) Fin length at 5 d.p.f. in the absence of wounding. (f) Images of 5 d.p.f.-larval tail fins with/without wounding. Note that wounding affects both the length and width of the wounded tail fins in plexin A1 morphants. (g) CRISPR-mediated plexin A1 inhibition impairs fin regeneration at 3 d.p.w. (5 d.p.f.) but not the fin length without wounding. ${ }^{\star} P<0.05$; one-way ANOVA with Dunnett's post-test. (h) Epithelial cell extrusion around a wound of Tg(cldnb:lynGFP). On average, $2.75(n=4$, s.e.m. $=0.48)$ cells were observed to be extruded for $5 \mathrm{~h}$ imaging, but note that all the extrusion events were not captured because imaging was done only in a part of the tail fin. (i) An extruded cell is undergoing apoptosis. (j) Representative images of transected tail fins at 9 h.p.w. (k) Knockdown of Plexin A1 increases apoptotic cells at wounds at 9 h.p.w. but not 1 h.p.w. ${ }^{\star} P<0.05$; one-way ANOVA with Dunnett's post-test.

UAS-Rap1 RNAi2 (BL35047)

UAS-Rap1 V12 (Hariharan lab, unpublished)

UAS-sema1a RNAi 1 (BL29554)

UAS-semala RNAi 2 (BL34320)

UAS-sema1b RNAi 1 (BL28588)

UAS-semalb RNAi 2 (NIG6446R-3)

UAS-HA-PlexA ${ }^{23}$ (a gift from Dr Terman and Dr Kolodkin)

UAS-HA-PlexA $\Delta$ cyto $^{23}$ (a gift from Dr Terman)

PlexA-myc $B A C^{40}$ (a gift from Dr Kolodkin)

UAS-p35 (BL5073)

UAS-Rok RNAi (VDRC104675)

UAS-dcr2; pnr-Gal4/TM3 (BL25758)

UAS-dcr2; Act5c-Gal4/Cyo (BL25708)

TRE reporter ${ }^{12}$ (a gift from Dr Bohmann)

Sp; Dr / SM5-TM6B

UAS-mCherry-moesin/Cyo

Pnr-Gal4/TM3 (BL3039)
E-Cad-GFP

For the TIE-DYE experiment, the following stocks were used as previously described ${ }^{41}$

y, w, hsFLP; Sp; Dr / SM5-TM6b

w Act $<$ stop $<$ lacz ${ }^{n l s}$, Ubi $<$ stop $<G F P^{n l s} ;$ Act $<$ stop $<$ gal4, UAS-

his $2 A:: R F P / S M 5-T M 6 b$

Using above stocks, the following stable stocks were built and used for experiments.

UAS-dcr2; nub-Gal4, UAS-GFP

UAS-dcr2; nub-Gal4, UAS-GFP, UAS-HA-PlexA

UAS-dcr2; nub-Gal4, UAS-GFP; UAS-PlexA RNAi 1

UAS-dcr2; nub-Gal4, UAS-GFP; UAS-PlexA RNAi 2

UAS-dcr2; nub-Gal4, UAS-GFP; UAS-sema1b RNAi 1

UAS-dcr2; nub-Gal4, UAS-GFP; UAS-sema1b RNAi 2

UAS-dcr2; nub-gal4, UAS-GFP; UAS-Rap2l RNAi 1

nub-Gal4; UAS-RFP

hsFLP;; UAS-PlexA RNAi 1 
hsFLP; UAS-HA-PlexA

hsFLP; UAS-HA-PlexA; UAS-GFP, Act $<$ stop $<$ gal4, UAS-GFP

UAS-mCherry-Moesin, E-Cadherin-GFP/Cyo; Pnr-Gal4/TM6b

In situ wound of wing discs. L3 larvae of the $n u b>G F P$ stock were quickly immobilized in ice water, and the right wing pouch was bisected by applying pressure on the larval cuticle with a thin and dull-pointed tungsten needle with the help of GFP fluorescence (see Supplementary Movie 1). This procedure does not involve penetration of the larval cuticle. For each fly stock, at least 25 larvae were wounded.

Calcium imaging. L3 larvae (nub-gal4/UAS-GCaMP3; UAS-RFP/+) were quickly immobilized in ice water and the right wing pouch was visualized using RFP and injured with a tungsten needle. Immediately after wounding, a larva was transferred on a slide glass and a coverslip was put on the larva to immobilize it completely. Live imaging of GCaMP3 fluorescence was performed with a stereomicroscope (Zeiss SteREO Lumar V12). Images were acquired every $10 \mathrm{~s}$. For kymographic analysis of a calcium flash, signals in a rectangular box were summed into a 1D line and kymograph was made with ImageJ (NIH).

Immunofluorescence and confocal imaging. Wing discs in late L3 larvae were dissected in PBS, fixed with paraformaldehyde in PBS and washed in $0.1 \%$ Triton in PBS. We used the following antibodies and fluorescent dyes: mouse $9 \mathrm{E} 10 \mathrm{myc}$ antibody (1:100, sc-40, Santa Cruz), rabbit cleaved Drosophila Dcp-1 antibody (1:400, \#9578, Cell Signaling), mouse anti-Arm (1:100, N2 7A1, DHSB), 4,6-diamidino-2-phenylindole (DAPI; 1:1,000, Molecular Probes), rhodamineconjugated phalloidin (1:500, Sigma-Aldrich) and Alexa Fluor 633 Phalloidin (1:50, Invitrogen). Fluorescence images were acquired with a confocal microscope (Zeiss LSM700). Three-dimensional reconstruction was performed by the Zen software. $z$ axis analysis was performed using Volocity (PerkinElmer). For the TIE-DYE clone analysis in Supplementary Fig. 3a,b, GFP signals that overlap with RFP signals were subtracted from the GFP channel to make the analysis simple. After the subtraction, clone sizes were quantified using ImageJ. For FRAP experiments, fluorescence of cadherin-GFP in $2-\mu \mathrm{m}$-diameter spots were bleached in the epithelia of the midline notum at 13 after puparium formation (APF) and images were acquired every $20 \mathrm{~s}$. Fluorescence recovery was quantified using ImageJ. For analysis of cell extrusion/delamination in the midline notum, timelapse images with Ecad-GFP were acquired in the midline of the notum from 13 to 25 APF. PlexA RNAi 1 was expressed in the notum using pnr-gal4.

Laser ablation and imaging. Flies with genotype w; UAS-mCherry-Moesin, E-Cadherin-GFP; pnr-GAL4/TM6b were crossed with PlexA RNAi 1 and kept at $29^{\circ} \mathrm{C}$. Pupae were staged at $13 \mathrm{~h}$ after puparium formation, and the notum epithelium was imaged at $29^{\circ} \mathrm{C}$ on an Andor Revolution XD spinning disk imaging system (Nikon Eclipse Ti-E and Yokogawa CSU-x1) with a Nikon (Plan Apo VC PFS) $\times 60$ oil-immersion objective. Pupae mounting was performed as previously described ${ }^{11}$. Wounds were made using a MicroPoint (Andor Technology) ultraviolet nitrogen-pumped dye laser $(435 \mathrm{~nm})-15$ pulses at maximum power. Images were acquired before and immediately after wounding every $2.5 \mathrm{~min}$ for $6 \mathrm{~h}$.

Wound area and junction quantification in Drosophila pupae. Wound area over time was quantified using Fiji manual selection tool and area measure function. Junction ratio (total junction length/vertex distance) was obtained by dividing the total membrane length value with the distance in a straight line between each end of the corresponding membrane vertex as shown in Fig. 2d. Measurements were done using five independent pupae for both the control and PlexA RNAi. At least 10 membranes from each pupa were randomly sampled and measured using the Fiji line segment tool. Data were processed in Microsoft Excel and GraphPad Prism 6.0 .

RT-PCR (Drosophila). Ten wing discs in late L3 larvae were dissected in PBS and snap-frozen at $-80^{\circ} \mathrm{C}$. RNA was prepared using the RNeasy Mini Kit (QIAGEN) and one-step RT-PCR (QIAGEN) was performed. To check efficiency of RNAi-mediated knockdown, wing discs in L3 larvae (UAS-Dcr2/ + or Y; Act5cgal4/UAS-RNAi or UAS-Dcr2/ + or Y; Act5c-gal4/ +; UAS-RNAi/ + ) were dissected. Oligonucleotide sequences for RT-PCR were as follows: $\alpha t u b 84 B$ forward, 5'-CCTTGTCGCGTGTGAAACACTTCC-3'; $\alpha t u b 84 B$ reverse, 5'-GTTGGCCGCATCCTCCTTACC-3';

PlexA forward, 5'-AAACATTCCATCAAGCCAAAGACCTCG-3'; PlexA reverse, $5^{\prime}$-TTAAGAGGACTTCCTGCGCGGC-3'; PlexB forward, 5'-AGACTTGGAAAAGATTCGCCGTCG-3'; PlexB reverse, 5'-GCAATCACCATCTAGTGTAGCAGC-3'; semala forward, 5'-ATACCAAAATGTGTCTCGTCAAGGGC-3'; semala reverse, $5^{\prime}$-CTTAGGCTATCGTACTGCTCGGTC- ${ }^{\prime}$; semalb forward, 5'-CGATGAGTTTGTTCTAGTTGGTGCC-3'; semalb reverse, 5'-CCTCCACATCTCGGCTGACTTC-3'; sema $a$ forward, $5^{\prime}$-GCAGTTTCCACATTAACGAGATTCAGG-3'; sema $2 a$ reverse, $5^{\prime}$-GACCCAGATTGGTGCCCACATAG-3'; $s e m a 2 b$ forward, $5^{\prime}$-TGCTGCCCATCATCTGCGCC-3'; $s e m a 2 b$ reverse, $5^{\prime}$-CGTTTACCCTTGGAAACACAATTCAGG- ${ }^{\prime}$; $s e m a 5 c$ forward, $5^{\prime}$-CGACAAAATCAAGTGTGGATTTGCTGG- $3^{\prime}$; sema $5 c$ reverse, $5^{\prime}$-CACGCGTACAGCTGGTTCTCAC-3'; MICAL forward, 5'-GACGCAGTGGAGACTTGCTGCC- $3^{\prime}$; $M I C A L$ reverse, 5'-GCAGATCTGGTCCAAATAGTTGAGCC-3';

Clone generation with heat shocks. Clones were induced by heat shocks in a $37-{ }^{\circ} \mathrm{C}$ circulating water bath. For experiments to make clones composed of multiple cells (Fig. 5d and Supplementary Figs 3a,b and 4a), a heat shock of 10 min was performed at $48-72 \mathrm{~h}$ after egg lay and the discs were dissected from late $\mathrm{L} 3$ larvae. For experiments to make single-cell clones (Fig. $4 \mathrm{j}-\mathrm{m}$ and Supplementary Fig. $4 \mathrm{~b}$ ), a heat shock of $14.5 \mathrm{~min}$ was performed at 100 -h after egg lay and the discs were dissected $10-12 \mathrm{~h}$ after the heat shock.

In vitro rap GAP assay. The active PlexA dimer construct was generated by fusing the GCN4 coiled-coil motif to the N terminus of the cytoplasmic region (residues 1,306-1,944) of Drosophila PlexA. The construct corresponds to the mouse $\mathrm{CC}(\mathrm{d}) \mathrm{PlexinA} 1_{\text {cyto }}$ as described previously, which displayed the highest RapGAP activity among all the tested dimer constructs ${ }^{25}$. The fusion was subcloned into a modified pET28 vector (Novagen) containing an N-terminal tandem $\mathrm{His}_{6}-\mathrm{SUMO}$ $\mathrm{tag}^{42}$. Drosophila Rap1 and Rap2l (residues 2-166 and 2-167, respectively) were subcloned into a modified pET28 vector containing an N-terminal $\mathrm{His}_{6}$ tag and a cleavage site for the $\mathrm{C} 3$ protease of human rhinovirus ${ }^{24,25}$. Proteins were expressed in the bacterial strain BL21 (DE3) and purified as previously described ${ }^{25}$. Removal of the SUMO and $\mathrm{His}_{6}$ tags was achieved by addition of the Ulp1 and C3 protease, respectively. Rap1 and 21 were GTP-loaded before use in the activity assays. GTP loading was achieved by incubating Rapl or 21 at a final concentration of $450 \mu \mathrm{M}$ over night at $4{ }^{\circ} \mathrm{C}$ in a buffer containing $9 \mathrm{mM}$ GTP, $3.4 \mathrm{mM}$ EDTA, $10 \mathrm{mM}$ Tris- $\mathrm{HCl} \mathrm{pH} \mathrm{8.0,10 \%} \mathrm{glycerol,} 150 \mathrm{mM} \mathrm{NaCl}$ and $2 \mathrm{mM}$ dithiothreitol. The exchange was quenched by the addition of $\mathrm{MgCl}_{2}$ to a final concentration of $20 \mathrm{mM}$. Removal of excess GTP was achieved by passing the mixtures over a size exclusion column (Superdex 75 10/300 GL, GE Healthcare). The GAP activity of the active dimer form of Drosophila PlexA measured using the photometric assay as describe by Webb and Hunter with minor modification $\mathrm{s}^{24,25,43}$. Briefly, the release of inorganic phosphate upon GTP hydrolysis is coupled to the reaction catalysed by purine nucleoside phosphorylase, which converts 2-amino-6-mercapto-7-methylpurine ribonucleoside (MESG) to its guanine base form (2-amino-6-mercapto-7-methylpurine) and ribose-1-phosphate in the presence of phosphate. MESG has a $\lambda_{\max }$ at $320 \mathrm{~nm}$, while the guanine base product has a $\lambda_{\max }$ at $360 \mathrm{~nm}$. The reaction was monitored spectroscopically by following the absorbance decay at $320 \mathrm{~nm}$ and the increase at $360 \mathrm{~nm}$ at $27^{\circ} \mathrm{C}$. PlexinA and Rap were mixed to final concentrations of 20 and $100 \mu \mathrm{M}$, respectively, in a reaction buffer containing $8 \mathrm{U} \mathrm{ml}^{-1} \mathrm{PNP}, 300 \mu \mathrm{M}$ MESG, $20 \mathrm{mM}$ Tris- $\mathrm{HCl} \mathrm{pH} \mathrm{7.6,} 20 \mathrm{mM} \mathrm{NaCl}, 5 \%$ glycerol, $2 \mathrm{mM}$ TCEP and $2 \mathrm{mM} \mathrm{MgCl}$. Each reaction was repeated three times. Owing to lack of the catalytic machinery, basal GTP hydrolysis of Rap1 and Rap2l in the absence of plexin is extremely slow and virtually undetectable under these condition $s^{24,25}$. Slow increases of the absorbance at $360 \mathrm{~nm}$ in reactions of either Rap1 or Rap2l without plexin were produced partially by spontaneous conversion of MESG. The absorbance increase was faster in reactions with Rap2l, which was because of precipitation of the protein, as indicated by the simultaneous increase at $320 \mathrm{~nm}$. To eliminate these artefacts, the plexin-catalysed rates were calculated by subtracting the slopes of the Rap-alone reactions from the slopes of the linear portions of the corresponding reactions in the presence of plexin.

Conditional gene inactivation by tissue-specific Cas9 expression. To induce wing-specific gene knockout, we generated flies that simultaneously carried the 11F02-GAL4 driver transgene ${ }^{44}$, a UAS-Cas9 transgene and a double-gRNA transgene. The UAS-Cas 9 transgene was generated in a custom UAS vector that has a $10 \times$ UAS sequence, gypsy insulators, a phiC31 attB sequence and a vermilion transgene. It was integrated into the attP40-landing site by phiC31mediated recombination ${ }^{45}$. Double-gRNA vectors were generated as described ${ }^{46}$. The gene-specific 20-bp sequences of gRNAs used in the present study are shown below for each target gene.

plexA: 5'-GAGCTGTAGTACGTTGTAGC-3' 5' $^{\prime}$-AATGCGAGAACGAGT TAATG-3'

e (ebony): 5'-GCCATCGTGCTCTACACCTC-3', 5'-GACTCTCAGGTGA AGATCCG-3'

$w g$ (wingless): $5^{\prime}$-GAGAAGGAAACAGCGACGCC-3', 5'-GGTGACCCA CTCGATTGCCA-3 $3^{\prime}$

ct $(c u t): 5^{\prime}$-GGACGAACTGCAGCGCTTGA-3', 5'-GAGCAGTGTGGCC CAGCATC- $3^{\prime}$

cv (crossveinless): $5^{\prime}$-GAAGAACTGCTCCTGCTGCA-3' ${ }^{\prime} 5^{\prime}$-GCTGTCCAA GAAGTCCCACG- $3^{\prime}$

Zebrafish maintenance and general procedures. Adult $\mathrm{AB}$ zebrafish and larvae were maintained according to the standard protocols ${ }^{47}$. For tail transection, 2-3 days post fertilization (d.p.f.) larvae were anaesthetized in E3 containing 
$0.2 \mathrm{mg} \mathrm{ml}^{-1}$ Tricaine (ethyl 3-aminobenzoate; Sigma-Aldrich) and the tail fin was resected with a razor blade ${ }^{2}$. Regenerate length was quantified by measuring the distance between the caudal tip of the notochord and the caudal tip of the tail fin at 3 days after wounding. For time-lapse imaging, tail fins of $T g(\operatorname{cldnb} \text { :lynGFP })^{48}$ were transected at 3 d.p.f. and time-lapse imaging was started immediately. Zebrafish immunostaining was performed as previously described ${ }^{2}$. A rabbit cleaved caspase3 antibody (1:300, \#9661, Cell Signaling) was used with DAPI (1:1,000, Molecular Probes) and rhodamine-conjugated phalloidin (1:500, Sigma-Aldrich).

MO injection and RT-PCR. MO oligonucleotides (Gene Tools) in Danieau buffer $\left(58 \mathrm{mM} \mathrm{NaCl}, 0.7 \mathrm{mM} \mathrm{KCl}, 0.4 \mathrm{mM} \mathrm{MgSO}_{4}, 0.6 \mathrm{mM} \mathrm{Ca}\left(\mathrm{NO}_{3}\right)_{2}\right.$ and $5.0 \mathrm{mM}$ HEPES, pH 7.1-7.3) were injected $(3 \mathrm{nl})$ into one-cell stage embryos. Danieau buffer was used as a control. For Plexin A3 knockdown, $500 \mu \mathrm{M}$ plexin A3 splice MO1 (5'-AGGCATGACATTGACTCACTTGTTA-3') or $500 \mu \mathrm{M}$ plexin A3 splice MO2 (5'-ATCTGAATGGTGTCTTACCATATCC- $3^{\prime}$ ) was used. For Plexin Al knockdown, $150 \mu \mathrm{M}$ plexin A1 splice MO1 (5'-AGCAGATAATTCTCTTACCGA GATC- $3^{\prime}$ ) or $150 \mu \mathrm{M}$ plexin A1 splice MO2 (5'-ACCAGTCGTTTGCTACCGTA CCTCC- $3^{\prime}$ ) was used. For ultraviolet-mediated Plexin A1 knockdown, combination of $471-\mu \mathrm{M}$ plexin A1 Photo-MO1 (5'-ATCTCGGTAAGPGAATTATCTGC$\left.3^{\prime}\right)$ and $429-\mu \mathrm{M}$ plexin $A 1$ splice MO1 or combination of $471 \mu \mathrm{M}$ plexin A1 PhotoMO2 (5'-GGTACGGTAPCAAACGACTGG-3') and $429-\mu \mathrm{M}$ plexin A1 splice MO2 was used. Photoactivation of Photo-MOs was performed with the Gene Tools UV light box (Gene Tools) in 1 d.p.f. embryos as described previously ${ }^{49}$. For morphotyping of the splicing MOs, RNA was prepared from 2.5 d.p.f. larvae using TRIZOL (Invitrogen), and one-step RT-PCR (QIAGEN) was performed.

Oligonucleotide sequences used for morphotyping RT-PCR were as follows:

plexin $A 1$ forward, 5'-GCTGCAGACTGATATCCATGAGC-3';

plexin A1 reverse, 5'-GTGACATCCTCGTCCTGGAG-3';

plexin A3 forward, 5'-TGAATGGTGTTCCTCCGCCG-3';

plexin $A 3$ reverse, $5^{\prime}$-TTGGACTTCAGAGAAAGCTTCACG-3'.

For expression analysis of plexins in larval tail fins, tail fins of 50 larvae at 3 d.p.f. were resected, RNA was prepared using TRIZOL and one-step RT-PCR was performed. Oligonucleotide sequences for RT-PCR were as follows: ef $1 \alpha$ forward, $5^{\prime}$-TACGCCTGGGTGTTGGACAAA-3'; ef $1 \alpha$ reverse, $5^{\prime}$-TCTTCTTGATGTATCCGCTGA- $3^{\prime}$; plexin A1 forward, 5'-GGAACGTGGCATCCCTCATC-3'; plexin A1 reverse, 5'-GTGACATCCTCGTCCTGGAG-3'; plexin A2 forward, 5'-GACTACTTTCCAACAATCTCCAGCCG-3'; plexin A2 reverse, 5'-CGCAGAAGCCATTATAGACATAGGAGG-3'; plexin A3 forward, 5'-GTAAACTGGTGGCGGATGAGG-3'; plexin A3 reverse, $5^{\prime}$-AACACCACCGAATGGTCTCCG-3'; plexin A4 forward, 5'-TTGTTCGTGGCCACAGCTGTTG-3'; plexin A4 reverse, 5'-GAGTGGTTCTTGTACACGTAGGC-3'.

CRISPR-Cas9 in zebrafish. We designed single guide RNAs (sgRNAs) by seeking sequences corresponding to $\mathrm{GGN}_{18} \mathrm{NGG}$ on the sense or antisense strand of plexin A1 DNA. To generate sgRNAs, we performed PCR with Phusion polymerase without a template ${ }^{50}$; a unique oligonucleotide encoding the T7 polymerasebinding site and the sgRNA target sequence $\mathrm{GGN}_{18} \mathrm{~N}$ (CRISPRF = GAAATTAAT ACGACTCACTATA GGN $_{18}$ GTTTTAGAGCTAGAAATAGC); and a common oligonucleotide encoding the remainder of the sgRNA sequence ( $\operatorname{sgRNAR}=\mathrm{AAA}$ AGCACCGACTCGGTGCCACTTTTTCAAGTTGATAACGGACTAGCCTTATT TTAACTTGCTATTTCTAGCTCTAAAAC). The DNA templates were used for in vitro transcription by T7 RNA polymerase (Ambion). Four unique sgRNAs were generated using the following CRISPRF oligonucleotides:

sgRNA 1, GAAATTAATACGACTCACTATAGG GAGATGGAGTACGCCA CTGTTTTAGAGCTAGAAATAGC;

sgRNA 2, GAAATTAATACGA CTCACTATAGGTAGCAAACGACTGGTT TAGTTTTAGAG CTAGAAATAGC:

sgRNA 3, GAAATTAATACGACTCACTAT AGGACAGAACCAAAGCGA GAGGTTTTA GAGCTAGAAATAGC

sgRNA 4,

GAAATTAATACGACTCACTATAGGACCGATCGACTCCATCACGTTTT AGAGCTAGAAATAGC

For making Cas9 RNA, pCS2-nCas9n (Addgene 47929) ${ }^{51}$ was linearized by Not1 and in vitro transcribed by SP6 RNA polymerase (Ambion). RNA of Cas9 $\left(250 \mathrm{ng} \mathrm{\mu l}^{-1}\right)$ and two sgRNAs (15 $\mathrm{ng}^{-1}$ each) in Danieau buffer were injected $(3 \mathrm{nl})$ into one-cell stage embryos. Cas9 only was used as a control.

Statistics. Figure 1i: ${ }^{\star} P<0.05$, two-tailed $\chi^{2}$-test with the Bonferroni correction (RNAi), two-tailed $\chi^{2}$-test (CRISPR), ctrl: $n=211$, PlexA RNAi 1: $n=90$, PlexA RNAi 2: $n=25$, PlexA RNAi 3: $n=20$, PlexA RNAi 4: $n=27$, Cas9: $n=98$, PlexA CRISPR: $n=39$.

Figure 2a: shadowed areas represent s.d. WT: $n=6$, PlexA RNAi: $n=3$.

Figure 2g: error bars represent s.e.m. ${ }^{*} P<0.05$; Kruskal-Wallis test with Dunn's post-test, WT before wound: $n=51$, PlexA RNAi before wound: $n=52$, WT after wound: $n=51$, PlexA RNAi after wound: $n=51$.

Figure 3a: ${ }^{\star} P<0.05$, two-tailed $\chi^{2}$-test with the Bonferroni correction, ctrl: $n=176$, PlexA RNAi 1: $n=44$, PlexA RNAi 2: $n=44$, semala RNAi 1, semalb
RNAi 1: $n=88$, semala RNAi 2, semalb RNAi 2: $n=53$, PlexA $\Delta$ cyto: $n=85$, MICAL RNAi: $n=150$, Rap2l RNAi 1: $n=68$, Rap2l RNAi $2: n=128$, Rap1 V12: $n=128$, Rap1 RNAi 1: $n=77$, Rap1 RNAi 2: $n=60$

Figure 4e: ${ }^{*} P<0.05$; two-tailed Mann-Whitney $U$-test, nub-gal4: 10 discs, 150 cells, nub-plexA RNAi: 10 discs, 166 cells.

Figure 4i: error bars represent s.e.m. (nub-PlexA: $n=6$, nub-PlexA, Rap1

RNAi: $n=6$ ).

Figure 41: error bars represent s.e.m. ( $n=17$ discs).

Figure $4 \mathrm{~m}$ : error bars represent s.e.m. (top: $n=5$, bottom: $n=6$ ).

Figure 5e: error bars represent s.e.m. (ctrl: $n=20$, PlexA RNAi: $n=18$ ).

Figure 6d: ${ }^{\star} P<0.05$; one-way analysis of variance (ANOVA) with Dunnett's

post-test, Ctrl: 15 larvae, MO1: 16 larvae, MO2: 18 larvae.

Figure 6g: ${ }^{*} P<0.05$; one-way ANOVA with Dunnett's post-test, Cas9,

+ wound: 38 larvae, Cas9, gRNA 1,2, + wound: 38 larvae, Cas9, gRNA 3,4,

+ wound: 42 larvae, Cas9, - wound: 25 larvae, Cas9, gRNA 1,2, - wound: 17

larvae, Cas9, gRNA 3,4, - wound: 17 larvae.

Figure 6k: ${ }^{\star} P<0.05$; one-way ANOVA with Dunnett's post-test, Ctrl 1 h.p.w.: 17 larvae, MO1: 1 h.p.w.: 12 larvae, MO2: 1 h.p.w.: 17 larvae, Ctrl 9 h.p.w.: 17 larvae, MO1: 9 h.p.w.: 14 larvae, MO2: 9 h.p.w.: 15 larvae.

Supplementary Fig. $3 \mathrm{~h}:{ }^{\star} P<0.05$, two-tailed $\chi^{2}$-test, + wound/ctrl: $n=99$,

+ wound/p35: $n=91,-$ wound/ctrl: $n=573$, - wound/p35: $n=310$.

Supplementary Fig. 5a: ${ }^{\star} P<0.05$, two-tailed unpaired $t$-test, pnr-gal4: $n=7$, pnr-PlexA RNAi: $n=6$.

Data availability. The data that support the findings and reagents used in this study are available from one of the corresponding authors (S.K.Y.) upon request.

\section{References}

1. Niethammer, P., Grabher, C., Look, A. T. \& Mitchison, T. J. A tissue-scale gradient of hydrogen peroxide mediates rapid wound detection in zebrafish. Nature 459, 996-999 (2009).

2. Yoo, S. K., Freisinger, C. M., LeBert, D. C. \& Huttenlocher, A. Early redox, Src family kinase, and calcium signaling integrate wound responses and tissue regeneration in zebrafish. J. Cell Biol. 199, 225-234 (2012).

3. Poss, K. D. Advances in understanding tissue regenerative capacity and mechanisms in animals. Nat. Rev. Genet. 11, 710-722 (2010)

4. Campos, I., Geiger, J. A., Santos, A. C., Carlos, V. \& Jacinto, A. Genetic screen in Drosophila melanogaster uncovers a novel set of genes required for embryonic epithelial repair. Genetics 184, 129-140 (2010).

5. Lesch, C., Jo, J., Wu, Y., Fish, G. S. \& Galko, M. J. A targeted UAS-RNAi screen in Drosophila larvae identifies wound closure genes regulating distinct cellular processes. Genetics 186, 943-957 (2010).

6. Losick, V. P., Fox, D. T. \& Spradling, A. C. Polyploidization and cell fusion contribute to wound healing in the adult Drosophila epithelium. Curr. Biol. 23, 2224-2232 (2013).

7. Tamori, Y. \& Deng, W. M. Tissue repair through cell competition and compensatory cellular hypertrophy in postmitotic epithelia. Dev. Cell 25, 350-363 (2013).

8. Tian, L. et al. Imaging neural activity in worms, flies and mice with improved GCaMP calcium indicators. Nat. Methods 6, 875-881 (2009).

9. Xu, S. \& Chisholm, A. D. A Galphaq-Ca $(2)(+)$ signaling pathway promotes actin-mediated epidermal wound closure in C. elegans. Curr. Biol. 21, 1960-1967 (2011).

10. Razzell, W., Evans, I. R., Martin, P. \& Wood, W. Calcium flashes orchestrate the wound inflammatory response through DUOX activation and hydrogen peroxide release. Curr. Biol. 23, 424-429 (2013).

11. Antunes, M., Pereira, T., Cordeiro, J. V., Almeida, L. \& Jacinto, A. Coordinated waves of actomyosin flow and apical cell constriction immediately after wounding. J. Cell Biol. 202, 365-379 (2013).

12. Chatterjee, N. \& Bohmann, D. A versatile PhiC31 based reporter system for measuring AP-1 and Nrf2 signaling in Drosophila and in tissue culture. PLoS ONE 7, e34063 (2012).

13. Galko, M. J. \& Krasnow, M. A. Cellular and genetic analysis of wound healing in Drosophila larvae. PLoS Biol. 2, E239 (2004)

14. Bosch, M., Serras, F., Martin-Blanco, E. \& Baguna, J. JNK signaling pathway required for wound healing in regenerating Drosophila wing imaginal discs. Dev. Biol. 280, 73-86 (2005).

15. Ramet, M., Lanot, R., Zachary, D. \& Manfruelli, P. JNK signaling pathway is required for efficient wound healing in Drosophila. Dev. Biol. 241, 145-156 (2002).

16. Kurusu, M. et al. A screen of cell-surface molecules identifies leucine-rich repeat proteins as key mediators of synaptic target selection. Neuron 59, 972-985 (2008).

17. Desai, R. et al. Monomeric alpha-catenin links cadherin to the actin cytoskeleton. Nat. Cell Biol. 15, 261-273 (2013).

18. Yu, J. A., Foley, F. C., Amack, J. D. \& Turner, C. E. The cell adhesion-associated protein Git2 regulates morphogenetic movements during zebrafish embryonic development. Dev. Biol. 349, 225-237 (2011). 
19. Tran, T. S., Kolodkin, A. L. \& Bharadwaj, R. Semaphorin regulation of cellular morphology. Annu. Rev. Cell Dev. Biol. 23, 263-292 (2007).

20. Winberg, M. L. et al. Plexin A is a neuronal semaphorin receptor that controls axon guidance. Cell 95, 903-916 (1998).

21. Yazdani, U. \& Terman, J. R. The semaphorins. Genome Biol. 7, 211 (2006).

22. Sweeney, L. B. et al. Temporal target restriction of olfactory receptor neurons by Semaphorin-1a/PlexinA-mediated axon-axon interactions. Neuron 53, 185-200 (2007)

23. Hung, R. J. et al. Mical links semaphorins to F-actin disassembly. Nature 463, 823-827 (2010).

24. Wang, Y. et al. Plexins are GTPase-activating proteins for Rap and are activated by induced dimerization. Sci. Signal. 5, ra6 (2012).

25. Wang, Y., Pascoe, H. G., Brautigam, C. A., He, H. \& Zhang, X. Structural basis for activation and non-canonical catalysis of the Rap GTPase activating protein domain of plexin. eLife 2, e01279 (2013).

26. Asha, H., de Ruiter, N. D., Wang, M. G. \& Hariharan, I. K. The Rap1 GTPase functions as a regulator of morphogenesis in vivo. EMBO J. 18, 605-615 (1999).

27. Knox, A. L. \& Brown, N. H. Rap1 GTPase regulation of adherens junction positioning and cell adhesion. Science 295, 1285-1288 (2002).

28. Wang, Y. C., Khan, Z. \& Wieschaus, E. F. Distinct Rap1 activity states control the extent of epithelial invagination via alpha-catenin. Dev. Cell 25, 299-309 (2013).

29. Pannekoek, W. J., Linnemann, J. R., Brouwer, P. M., Bos, J. L. \& Rehmann, H. Rap1 and Rap2 antagonistically control endothelial barrier resistance. PLoS ONE 8, e57903 (2013)

30. Mizumoto, K. \& Shen, K. Interaxonal interaction defines tiled presynaptic innervation in C. elegans. Neuron 77, 655-666 (2013).

31. Chera, S. et al. Apoptotic cells provide an unexpected source of Wnt3 signaling to drive hydra head regeneration. Dev. Cell 17, 279-289 (2009).

32. Tseng, A. S. \& Levin, M. Tail regeneration in Xenopus laevis as a model for understanding tissue repair. J. Dental Res. 87, 806-816 (2008).

33. Nakajima, Y., Meyer, E. J., Kroesen, A., McKinney, S. A. \& Gibson, M. C. Epithelial junctions maintain tissue architecture by directing planar spindle orientation. Nature 500, 359-362 (2013).

34. Eisenhoffer, G. T. \& Rosenblatt, J. Bringing balance by force: live cell extrusion controls epithelial cell numbers. Trends Cell Biol. 23, 185-192 (2013).

35. Sawyer, J. K., Harris, N. J., Slep, K. C., Gaul, U. \& Peifer, M. The Drosophila afadin homologue Canoe regulates linkage of the actin cytoskeleton to adherens junctions during apical constriction. J. Cell Biol. 186, 57-73 (2009).

36. Ando, K. et al. Rap1 potentiates endothelial cell junctions by spatially controlling myosin II activity and actin organization. J. Cell Biol. 202, 901-916 (2013).

37. Koto, A., Kuranaga, E. \& Miura, M. Apoptosis ensures spacing pattern formation of Drosophila sensory organs. Curr. Biol. 21, 278-287 (2011).

38. Marinari, E. et al. Live-cell delamination counterbalances epithelial growth to limit tissue overcrowding. Nature 484, 542-545 (2012).

39. Xia, J. et al. Semaphorin-Plexin signaling controls mitotic spindle orientation during epithelial morphogenesis and repair. Dev. Cell 33, 299-313 (2015).

40. Pecot, M. Y. et al. Multiple interactions control synaptic layer specificity in the Drosophila visual system. Neuron 77, 299-310 (2013).

41. Worley, M. I., Setiawan, L. \& Hariharan, I. K. TIE-DYE: a combinatorial marking system to visualize and genetically manipulate clones during development in Drosophila melanogaster. Development 140, 3275-3284 (2013).

42. He, H., Yang, T., Terman, J. R. \& Zhang, X. Crystal structure of the plexin A3 intracellular region reveals an autoinhibited conformation through active site sequestration. Proc. Natl Acad. Sci. USA 106, 15610-15615 (2009).

43. Webb, M. R. \& Hunter, J. L. Interaction of GTPase-activating protein with p21ras, measured using a continuous assay for inorganic phosphate release. Biochem. J. 287(Pt 2): 555-559 (1992).

44. Jory, A. et al. A survey of 6,300 genomic fragments for cis-regulatory activity in the imaginal discs of Drosophila melanogaster. Cell Rep. 2, 1014-1024 (2012).
45. Markstein, M., Pitsouli, C., Villalta, C., Celniker, S. E. \& Perrimon, N. Exploiting position effects and the gypsy retrovirus insulator to engineer precisely expressed transgenes. Nat. Genet. 40, 476-483 (2008)

46. Kondo, S. \& Ueda, R. Highly improved gene targeting by germline-specific Cas 9 expression in Drosophila. Genetics 195, 715-721 (2013).

47. Nusslein-Volhard, C. \& Dahm, R. Zebrafish: a Practical Approach 1st edn (Oxford University Press, 2002).

48. Haas, P. \& Gilmour, D. Chemokine signaling mediates self-organizing tissue migration in the zebrafish lateral line. Dev. Cell 10, 673-680 (2006)

49. Tallafuss, A. et al. Turning gene function ON and OFF using sense and antisense photo-morpholinos in zebrafish. Development 139, 1691-1699 (2012).

50. Bassett, A. R., Tibbit, C., Ponting, C. P. \& Liu, J. L. Highly efficient targeted mutagenesis of Drosophila with the CRISPR/Cas9 system. Cell Rep. 4, 220-228 (2013).

51. Jao, L. E., Wente, S. R. \& Chen, W. Efficient multiplex biallelic zebrafish genome editing using a CRISPR nuclease system. Proc. Natl Acad. Sci. USA 110, 13904-13909 (2013).

\section{Acknowledgements}

We thank D. Bohmann, J. Knoblich, A. Kolodkin, J. Terman and TRiP at Harvard Medical School, the Bloomington Stock Center, the Vienna Drosophila RNAi center and NIG for transgenic fly stocks. We thank J. Bosch for sharing RNAi stocks, other Hariharan laboratory members for help with fly work, S. Amacher and N. Shih for help with zebrafish work and D. Bilder, R. Harris, N. Patel and M. Worley for comments on the manuscript. S.K.Y. was supported by the Miller Institute; I.K.H. by R01GM085576 from NIGMS and an American Cancer Society Research Professor Award 120366-RP-11-07801-DDC; X.Z. and H.G.P. by GM088197 from NIGMS and I-1702 from the Welch Foundation; A.J. and T.P. by European Research Council (2007-StG-208631); and S.K. by Mochida Memorial Foundation for Medical and Pharmaceutical Research.

\section{Author contributions}

S.K.Y. and I.K.H. conceived and oversaw the project. S.K.Y., H.G.P. and T.P. executed the experiments: S.K.Y. executed most experiments except laser wounding in Drosophila pupae (T.P.) and GAP assays (H.G.P.). H.G.P. and X.Z. contributed expertise in protein purification and in vitro GAP assays. T.P. and A.J. contributed expertise in laser wounding in Drosophila pupae. S.K. contributed expertise and reagents in Drosophila CRISPR. S.K.Y., T.P., S.K., X.Z. and I.K.H. wrote the paper.

\section{Additional information}

Supplementary Information accompanies this paper at http://www.nature.com/ naturecommunications

Competing financial interests: The authors declare no competing financial interests.

Reprints and permission information is available online at http://npg.nature.com/ reprintsandpermissions/

How to cite this article: Yoo, S. K. et al. Plexins function in epithelial repair in both Drosophila and zebrafish. Nat. Commun. 7:12282 doi: 10.1038/ncomms12282 (2016)

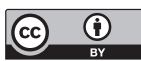

This work is licensed under a Creative Commons Attribution 4.0 International License. The images or other third party material in this article are included in the article's Creative Commons license, unless indicated otherwise in the credit line; if the material is not included under the Creative Commons license, users will need to obtain permission from the license holder to reproduce the material. To view a copy of this license, visit http://creativecommons.org/licenses/by/4.0/

(C) The Author(s) 2016 